\title{
Control of mitochondrial superoxide production includes programmed mtDNA deletion and restoration
}

Authors: Simon Stenberg ${ }^{1,2}$, Jing $\mathrm{Li}^{3,4}$, Arne B. Gjuvsland ${ }^{1}$, Karl Persson ${ }^{2}$, Erik Demitz-Helin ${ }^{2}$, Jia-Xing Yue ${ }^{3,4}$, Ciaran Gilchrist ${ }^{2}$, Timmy Ärengård ${ }^{2}$, Payam Ghiaci², Lisa Larsson-Berglund ${ }^{2}$, Martin Zackrisson ${ }^{2}$, Johanna L. Höög ${ }^{2}$, Mikael Molin ${ }^{2,5}$, Gianni Liti ${ }^{4}$, Stig W. Omholt ${ }^{6 *}$, Jonas Warringer ${ }^{2 *}$

\section{Affiliations:}

${ }^{1}$ Centre for Integrative Genetics (CIGENE), Department of Animal and Aquacultural Sciences, Norwegian University of Life Sciences, PO Box 5003, 1432 Ås, Norway 2 Department of Chemistry and Molecular Biology, University of Gothenburg, PO Box 462, 40530 Gothenburg, Sweden

${ }^{3}$ State Key Laboratory of Oncology in South China, Collaborative Innovation Center for Cancer Medicine, Sun Yat-sen University Cancer Center, Guangzhou, China.

${ }^{4}$ University of Côte d'Azur, CNRS, INSERM, IRCAN, Nice, France

5 Department of Biology and Biological Engineering, Chalmers University of Technology

${ }^{6}$ Department of Circulation and Medical Imaging, Cardiac Exercise Research Group, Norwegian University of Science and Technology (NTNU), 7491, Trondheim, Norway

*Corresponding authors: jonas.warringer@cmb.gu.se; stig.omholt@ntnu.no 


\section{SUMMARY}

Age-related diseases are intimately linked to mitochondrial impairment. Whether oxidative stress is a major driver of this impairment is still a contentious issue. Here we show that yeast cells adapt to intramitochondrial superoxide anion $\left(\mathrm{O}_{2}^{-}\right)$production beyond antioxidant defenses by swiftly reducing the copy numbers of mitochondrial electron transport chain (ETC) genes, while maintaining the copy numbers of undeleted mtDNA. The copy numbers of the ETC genes are rapidly restored after cessation of a short-term stress, whereas long-term stress causes irreversible loss of this capacity through maladaptive persistence of the mtDNA deletion process. As chronic oxidative stress is a hallmark of ageing, maladaptive mtDNA deletion may be a marked contributor to age-related mtDNA impairment.

\section{KEYWORDS}

Mitochondria, Oxidative stress, Aging, Yeast

\section{INTRODUCTION}

Although involved in many aspects of anabolism and cellular homeostatic regulation, a primary role of mitochondria is to produce adenosine triphosphate (ATP) by the mitochondrial electron transport chain (ETC). Reactive oxygen species (ROS) are generated through premature electron leakage from at least 11 different sites associated with substrate catabolism and the electron transport chain (Brand, 2016). The primary ROS formed in the mitochondrial matrix is superoxide radical anion $\left(\mathrm{O}_{2}^{-}\right)$, and the major fraction of $\mathrm{O}_{2}^{-}$emerges from the biochemical activities of 
complex I, complex III, and the leakage of electrons from carriers associated with these complexes (Zou et al., 2017).

Under normal physiological conditions, the intramitochondrial $\mathrm{O}_{2}^{-}$level is strictly regulated to control oxidative stress (Sies, 2018). When the production of $\mathrm{O}_{2}^{-}$ exceeds the capacity of this regulation, the mitochondrial matrix starts to experience oxidative stress, characterized by breakdown of redox signaling and accumulation of molecular damage. The current conception is that such a supraphysiological $\mathrm{O}_{2}^{-}$level evokes the mitochondrial unfolded protein response $\left(U P R^{\mathrm{mt}}\right)$ that includes an enhanced antioxidant defense (Shpilka \& Haynes, 2018). In the event that this regulation fails to recover mitochondrial integrity and the oxidative damage reaches a threshold, a mitophagic response is launched (Bess, Crocker, Ryde, \& Meyer, 2012; Gustafsson \& Dorn, 2019; Lemasters, 2005; Palikaras \& Tavernarakis, 2014; Sedlackova \& Korolchuk, 2019). However, it is not yet known whether mitophagy is the only way eukaryotic cells cope with an intramitochondrial supraphysiological $\mathrm{O}_{2}^{-}$ challenge overwhelming the capacity of the antioxidant defense system. Thus, we cannot exclude the possibility that we are currently neglecting regulatory mechanisms associated with oxidative stress that are instrumental for understanding why mitochondrial impairment is so strongly associated with ageing (Sun, Youle, \& Finkel, 2016) and the pathogenesis of age-related human diseases, including Alzheimer's disease (Hu, Tan, Tan, \& Yu, 2017), Parkinson's disease (Ammal Kaidery \& Thomas, 2018), the deterioration of skeletal and cardiac muscle (Hepple, 2016), and macular degeneration (Hyttinen, Viiri, Kaarniranta, \& Błasiak, 2018).

An effectual way to test whether mitotic eukaryotic cells possess undisclosed mechanisms controlling intramitochondrial $\mathrm{O}_{2}^{-}$levels overwhelming the antioxidant defense system is to make use of an experimental evolution framework wherein the 
adaptive responses to such stress can be systematically scrutinized. Applying this experimental paradigm to clonally expanding budding yeast (Saccharomyces cerevisiae), we found that the first-line response of budding yeast to this type of intramitochondrial oxidative stress is to invoke a programmed adaptation that very quickly and very consistently deletes mtDNA encoding electron transport chain proteins in a targeted manner. If the stress is sustained, the second-line response is a genetic adaptation by duplication of specific chromosomes that reduces the deleterious effects of mitochondrial $\mathrm{O}_{2}^{-}$further. Chronic stress causes a sustained maladaptive operation of the ETC mtDNA deletion mechanism that subsequently leads to irrevocable mitochondrial impairment through complete annihilation of the heteroplasmic state. We see no apparent reason for why the observed adaptive and maladaptive responses would not extend to postmitotic cells. The results therefore appear to strengthen the case for oxidative stress being a major etiological agent of age-related diseases.

\section{RESULTS}

\section{Early $\mathbf{O}_{2}^{-}$Adaptation is Under Regulatory Control}

We exposed haploid yeast populations expanding clonally on glucose to the mitochondrial $\quad \mathrm{O}_{2}^{-} \quad$ generator paraquat $\quad(\mathrm{N}, \mathrm{N}$-dimethyl-4-4'-bipiridinium dichloride)(Cochemé \& Murphy, 2008) in order to induce an elevated intramitochondrial $\mathrm{O}_{2}^{-}$level (Figure 1A). As electron transport chain (ETC) complex III and mitochondrial NADPH dehydrogenases donate electrons to paraquat, which passes these on to $\mathrm{O}_{2}$ (Castello, Drechsel, \& Patel, 2007; Cochemé \& Murphy, 2008), this mode of $\mathrm{O}_{2}^{-}$generation is a good proxy for the in vivo situation (Zou et al., 2017). We wanted to mimic a supraphysiological oxidative challenge qualifying as 
oxidative stress well beyond the regulatory reach of the mitochondrial antioxidant defense machinery, while not severely compromising cellular function. To this end, we titrated the paraquat dose such that the levels of transcripts encoding the antioxidant defense proteins Sod1, Sod2 and Ccp1 were markedly enhanced (2-12 fold in early lag-phase) but still clearly incapable of maintaining a normal cell doubling time (2.5-3 fold increase in cell doubling time). Cells maintained the enhanced expression of these transcripts throughout the exponential growth phase and the following two batch-cultivation cycles (Figure 1B), without causing a notable change in cell doubling time. The mitochondrial superoxide defense system remained elevated during the first hours after release from $\mathrm{O}_{2}^{-}$stress.

We then measured how fast 1152 clonal yeast populations adapted to the selected paraquat dose compared to those of in total $>8000$ populations exposed to seven other stressors not explicitly challenging mitochondrial function (Table S1). A chronological record of 96 random cell populations per challenge (Figure S1) was frozen for later revival and reanalysis. From these 768 (96x8) populations we extracted the number of cell divisions and the associated cell doubling time from each batch cycle to generate exact adaptation profiles.

All populations exposed to paraquat adapted much faster than every other population exposed to other stressors (Figure 1C; Figure S2A). The early adaptation response to paraquat was both very swift and substantial. Within the first 10 generations, the populations reduced their cell doubling time by 106 min, corresponding to $49.3 \%$ of the maximally achievable reduction. Thereafter, the adaptation slowly progressed before levelling off at $72.6 \%$ of the maximally achievable reduction in cell doubling time after 75 generations (mean), defining a 
second adaptation phase that was distinctly different from the first. Acknowledging that fast adaptation in haploid budding yeast may in rare cases be achieved by a purely Darwinian process of random mutations in the nuclear DNA and subsequent selection (Gjuvsland et al., 2016), we assessed this possibility by use of a Darwinian evolutionary model combining population genetics, dynamics and data on mutation rates and effect sizes of de novo point mutations and aneuploidies. The model was unable to reproduce the observed consistent and extraordinary swift response to $\mathrm{O}_{2}^{-}$stress (Figure 1D), further supporting that the observed early phase rate of reduction in cell doubling time could not be reconciled with pure random mutations in the nuclear DNA and subsequent selection process.

To assess how fast the acquired adaptation was lost after removal of a shortterm oxidative stress, we released each of the 96 frozen cell populations, at multiple stages of adaptation, from paraquat exposure for 10 batch cycles (mean of 84 generations). After each cycle, we re-exposed populations to the same $\mathrm{O}_{2}^{-}$stress, tracking the loss of their acquired reduction in cell doubling time (adaptation gain) as a function of the number of generations post- $\mathrm{O}_{2}^{-}$stress. All cell populations released from selection during the first phase of $\mathrm{O}_{2}^{-}$adaptation retained their adaptation gains over 1-2 batch cycles, before abruptly losing them (Figure 1E). The growth of the adapted cell populations was on par with that of founder populations in absence of $\mathrm{O}_{2}^{-}$stress (Figure $1 \mathrm{~F}$ ), demonstrating that the abrupt and consistent loss of their adaptation gains occurred despite no or marginal Darwinian counterselection. In stark contrast, cell populations from the other environments to which adaptation was also fast (arsenic and glycine) lost their adaptation gains progressively (Figure S2B) despite the presence of much stronger Darwinian counterselection (Figure S2C). 
The experimental data therefore strongly suggest that yeast cells indeed possess a highly efficient programmed adaptation for handling intramitochondrial supraphysiological $\mathrm{O}_{2}^{-}$stress beyond the mitochondrial antioxidant defense system.

\section{Early $\mathbf{O}_{2}^{-}$Adaptation Involves Specific Deletion of ETC Genes}

As mitochondrial $\mathrm{O}_{2}^{-}$generation is known to influence the mitochondrial fission and fusion dynamics (Frank et al., 2012; Hung et al., 2018), and mitochondrial fragmentation is a well-documented prelude to mitophagy (Sprenger \& Langer, 2019), we assayed mitochondrial morphology in pre- and post- $\mathrm{O}_{2}^{-}$exposed cells by use of confocal and electron microscopy. We observed a rapid $(<5 \mathrm{~h})$ shift from a tubular to a fragmented mitochondrial organization (Figures 2A and 2B; Figure S3A), and virtually all fragments contained mtDNA (Figure S3B). The fragmented mitochondrial morphology rapidly $(<5 \mathrm{~h})$ reverted back to a tubular organization after removal of $\mathrm{O}_{2}^{-}$stress, underscoring that the morphological dynamics was under strict regulatory control also during recovery from $\mathrm{O}_{2}^{-}$stress. The mitochondrial fragmentation did not lead to any loss of mitochondrial volume, even after $77 \mathrm{~h}$ of paraquat exposure (Figure 2B; right diagram), arguing against the operation of a canonical mitophagic response.

Guided by the observation that mitochondrial fragmentation promotes selective removal of deleterious mtDNA in the germline (Lieber, Jeedigunta, Palozzi, Lehmann, \& Hurd, 2019), the observed lack of a clear canonical mitophagic response led us to suspect that the fast adaptation to paraquat was due to change of mitochondrial function. We therefore assayed the growth of populations at different adaptation stages on a respiratory growth medium (glycerol) and found that the early $\mathrm{O}_{2}^{-}$adaptation correlated perfectly with a marked loss of respiratory growth (Figure 
$3 \mathrm{~A})$. Moreover, the swift loss of $\mathrm{O}_{2}^{-}$adaptation following a shift back to a normal growth medium coincided with the recovery of respiratory growth (Figure 3B), which indicated that the first $\mathrm{O}_{2}^{-}$adaptation phase involved loss of ETC function. We therefore measured the mtDNA content in five random $\mathrm{O}_{2}^{-}$exposed populations by genome sequencing, finding a clear inverse temporal association between the median mtDNA coverage and adaptation gain (Figure $3 C$ ). While the mtDNA sequence coverage after the first 3-4 generations of $\mathrm{O}_{2}^{-}$exposure remained uniform (Figure S3C), quantitative PCR showed that one or more segments within the mtDNA region spanning COX1 to $V A R 1$, and in rare cases also encompassing 21S RNA and COX2 (Figure 3D; Figure S4), was rapidly lost thereafter. Cells retained the remaining mtDNA segments, which always included COX3-RPM1 and 15S RNA, at near founder levels throughout the first $\mathrm{O}_{2}^{-}$adaptation phase. This strongly suggested that targeted deletion of ETC genes caused the observed loss of capacity for respiratory growth and the marked early-phase adaptation gain.

To confirm that restoration of ETC genes accounted for the reestablishment of respiratory growth after removal of $\mathrm{O}_{2}^{-}$stress, we repeated the $\mathrm{O}_{2}^{-}$relaxation experiment on the five sequenced populations. As anticipated, the swift restoration of respiratory growth post short-term $\mathrm{O}_{2}^{-}$stress was concomitant with the restoration of mtDNA copy numbers of deleted ETC genes back to pre-stress levels (Figures 3E and $3 F)$.

Considering that $68 \%$ of the realized adaptation on paraquat was associated with the early mtDNA deletions, the major fraction of paraquat-induced $\mathrm{O}_{2}^{-}$production is arguably associated with the ETC (Castello et al., 2007). And it suggests that respiration is far from being totally repressed (Crabtree, 1929) in cells growing on glucose in an aerobic environment, which is fully in line with the observation that $\rho^{0}$ 
cells devoid of mtDNA under these conditions show a 2-fold decrease in cellular $\mathrm{O}_{2}^{-}$ production (Reddi \& Culotta, 2013).

The main conclusion from the above experimental data is that yeast cells possess a programmed adaptation to supraphysiological intra-mitochondrial $\mathrm{O}_{2}^{-}$ stress that operates by deleting and restoring ETC-encoding mtDNA segments. As neither antioxidant defense regulation nor canonical mitophagic regulation appeared to contribute to the fast adaptive response, the adaptation qualifies as a distinct regulatory entity.

\section{Chromosome Duplications Explain the Second Adaptation Phase}

The fact that the cells realized $23.3 \%$ of their adaptation potential in the first 65 generations of the second adaptation phase (Figure 1C) demonstrated that the cells continued to sense and adapt to increased mitochondrial $\mathrm{O}_{2}^{-}$. To search for a molecular explanation of this second adaptation phase, we sequenced 44 random endpoint $\left(t_{50}\right)$ populations. Nuclear point mutations neither recurred in the same genes across populations (Figure S5A) nor coincided in time with $\mathrm{O}_{2}^{-}$adaptation (Figure S5B) in the 44 random endpoint $\left(t_{50}\right)$ populations. These mutations were therefore unlikely to have played a prominent causative role in the adaptation process. However, all but 4 endpoint populations carried extra chromosome II ( $n=29)$, III $(n=21)$ and/or $\mathrm{V}(n=16)$ copies (Figure 4A) at near fixation (mean $p: 0.97)$. These chromosome gains succeeded the first $\mathrm{O}_{2}^{-}$adaptation phase (Figure S5C). To assess their contribution to the second phase of adaptation, we backcrossed clones carrying the individual aneuploidies to wildtype cells and compared $\mathrm{O}_{2}^{-}$tolerance and capacity for respiratory growth in third generation meiotic progeny with extra chromosomes to those without. We found that chromosome II or $\mathrm{V}$ duplications 
reduced the cell doubling time during $\mathrm{O}_{2}^{-}$exposure by 31 and $38 \mathrm{~min}$, respectively $(s=0.11$ and 0.12$)$ (Figure 4B). Assuming an additive phenotypic effect of these duplications, the cell doubling time would be reduced by $69 \mathrm{~min}$. Together with the effect from ETC mtDNA deletion, this corresponds to a $81 \%$ realization of the adaptation potential. As the cells realized on average $72.6 \%$, an approximately additive phenotypic effect of these duplications appears to fully explain the second phase of adaptation in populations having fixed both these duplications.

\section{Chronic $\mathrm{O}_{2}^{-}$Stress Causes Irreversible Mitochondrial Impairment by Sustained mtDNA Deletion}

We found that the capacity to restore copy numbers of intact mtDNA and respiratory growth (Figure 5A) after removal of $\mathrm{O}_{2}^{-}$stress was lost in the five focal cell populations between 15 to 42 generations of paraquat exposure, coinciding with genetic fixation of the $\mathrm{O}_{2}^{-}$adapted phenotype (Figure 1E). In the larger set of 96 frozen fossil populations, $46 \%$ of the populations showed fixation after 24 generations, and after 242 generations all did. This led us to probe how persistent oxidative stress could cause this irrevocable mitochondrial impairment.

Out of the 44 sequenced endpoint $\left(t_{50}\right)$ populations exposed to chronic $\mathrm{O}_{2}^{-}$ stress, 24 had lost almost completely the entire $77 \mathrm{~kb}$ mtDNA (97-99\%) and contained only reads for small mtDNA segments $(<1-2 \mathrm{~kb})$ (Figure 5B; Figure S6A). We stained the DNA in these cells to confirm that they retained small mtDNA segments and dubbed them rho hypernegatives $\left(\rho^{--}\right)$to distinguish them from rho null $\left(\rho^{0}\right)$ ones (Figure S6B). Because some of these cell populations had retained much larger mtDNA segments throughout the first adaptation phase, their rho hypernegative state emerged only after extended $\mathrm{O}_{2}^{-}$stress. Thus, these additional 
mtDNA deletions occurred after key ETC genes were already removed. 18 endpoint populations remained in a rho negative $\left(\rho^{-}\right)$states and still possessed 6 to $34 \mathrm{~kb}$ mtDNA segments with copy numbers somewhat above founder levels (mean: $20 \%$ increase). All of these rho negative populations lacked $C O B$, while only 6 lacked $R P M 1$, the rRNA subunit of mitochondrial RNase $\mathrm{P}$ (Figure $5 \mathrm{C}$ ), underscoring that their mtDNA loss was non-random. A single population (A12) retained an intact mtDNA profile, but at copy numbers below founder levels (mean: $34 \%$ coverage reduction) (Figure 5B, upper panel; see Methods for further details).

Chromosome duplications contributed only marginally ( 2.4 vs $1.6 \mathrm{~h}$ for the founder) to the complete loss ( $>24 \mathrm{~h}$ doubling time) of respiratory growth (Figure $\mathrm{S6C)}$. Thus, as neither nuclear point mutations nor aneuploidy appeared capable of explaining the constitutive loss of restorative capacity, irrevocable loss of ETC encoding mtDNA emerged as the determinative causative factor. The loss of restorative capacity therefore appears to be caused by annihilation of the heteroplasmic state where intact mtDNA genomes are still present. Furthermore, the most parsimonious explanation for the observed irreversible loss of almost the entire $77 \mathrm{~kb} \mathrm{mtDNA}$ in more than $50 \%$ of the $t_{50}$ populations is that the cells possess no regulatory brake that prevents the almost complete depletion of mtDNA as long as the oxidative stress is sustained. To find experimental support for this we compared the fitness of the $\rho^{-}$and $\rho^{--}$populations. The $\rho^{--}$populations became less fit than the $\rho^{-}$ones during the second phase of adaptation (Figure 5D).

The data imply that mtDNA loss beyond a certain stage does not emerge by a Darwinian adaptive process, but by a maladaptive response driven by prolonged induction of a regulatory program dimensioned by natural selection to handle $\mathrm{O}_{2}^{-}$ 
stress challenges it can successfully deal with before the heteroplasmic state is annihilated (Figure 6).

\section{DISCUSSION}

The maintenance of homeostatic levels of non-targeted mtDNA under temporary $\mathrm{O}_{2}^{-}$stress suggests that ETC mtDNA deprived genomes were replicated along with intact mtDNA genomes, and that removal of these genomes was deliberately repressed as long as the stress prevailed. The capacity to restore the intact mtDNA genome pool back to pre-stress levels after cessation of a short-term stress suggests that the restoration process involves deliberate release of this repression and subsequent removal of ETC mtDNA deprived genomes. Thus, the disclosed genetically programmed adaptation appears to encompass two distinct, but integrated, regulatory schemes.

\section{Possible Mechanisms Underlying mtDNA Deletion}

We do not know the molecular mechanisms responsible for the observed mtDNA deletions. It is conceivable that $\mathrm{O}_{2}^{-}$stress induces mitochondrial genomic instability that leads to mtDNA deletions and subsequent adaptation gains through selection of those cells that have preferentially deleted ETC mtDNA segments. However, the copy number homeostasis of non-targeted segments across populations in the early adaptation phase speaks strongly against the operation of random $\mathrm{O}_{2}^{-}$-induced mtDNA deletions, as this would imply specific selection against the deletion of such segments. The data clearly show that this is not the case after the first adaptation phase. Thus, to achieve the selectivity observed in the first adaptation phase, natural selection would have to have built in genetically programmed means to achieve non- 
random deletions. The observed replication of ETC mtDNA deprived genomes until cessation of stress, and their subsequent rapid replacement by intact mtDNA, also implies the presence of deliberate control. But this does not preclude that selection contributes to the first adaptation phase to some degree.

Another explanatory option follows from the recent observation that Poly exonuclease activity declined more rapidly than polymerase activity under oxidative stress, causing the oxidized Poly to become editing-deficient (Anderson, Luo, Russell, \& Yin, 2020). This is fully in line with the observation that inactivation of the yeast ortholog Mip1 exonuclease activity results in a 160-fold increase of the frequency of deletions between 21 bp direct repeats (Stumpf \& Copeland, 2013). Inactivation of Poly exonuclease activity appears to cause double-strand breaks (DSBs) (Macao et al., 2015) and repair of these by microhomology-mediated end joining (MMEJ) is prone to cause deletions (Tadi et al., 2016). It is therefore not inconceivable that a Poly-MMEJ dependent process is involved in generating the $\mathrm{O}_{2}^{-}$induced mtDNA deletions. However, this mechanism does not readily explain the specificity of the early mtDNA deletions unless one also in this case invokes a genetically programmed mechanism ensuring this specificity. A combination of a genomic instability and a Poly-MMEJ process or some other process can neither be excluded.

\section{Possible Mechanisms Underlying mtDNA Restoration}

Even though we do not know the exact molecular mechanisms responsible for the observed restoration of the intact mtDNA pool after release from paraquat, the data provide some clues. Because restoration of intact mtDNA genomes back to prestress level is almost complete after just 2-3 batch cycles (Figure 3F) and 
because this happens after the mitochondria have returned to a tubular morphology (Figure 3B), it is hard to conceive how it can be achieved by asymmetric inheritance of mitochondria between mother and daughter cells during cellular replication (Katajisto et al., 2015; Vevea, Swayne, Boldogh, \& Pon, 2014). This points to the operation of a mitophagic process specifically targeting removal of ETC mtDNA deprived genomes not dependent on a fully fragmented mitochondrial morphology.

The $\mathrm{K}^{+}$-ionophore valinomycin induces overproduction of mitochondrial ROS and subsequent mitochondrial damage in yeast (Andrukhiv, Costa, West, \& Garlid, 2006; Selivanov et al., 2008). This rapidly leads to formation of Atg11-containing autophagosomal structures at the mitochondrial surface, and these structures are instrumental for selective removal of complex I and complex III subunits (TimónGómez, Sanfeliu-Redondo, Pascual-Ahuir, \& Proft, 2018). This is reminiscent of the mitochondria-derived vesicles (MDV) mechanism, which in mammals is responsible for local mitochondrial repair based on the mitochondrial export of 70 to $150 \mathrm{~nm}$ single- or double-membraned vesicles containing mitochondrial cargo that bud from the outer mitochondrial membrane, and which are subsequently degraded (Gustafsson \& Dorn, 2019). This provides tentative support for the notion that yeast possess a regulatory system for local mitochondrial repair that is deliberately repressed while the ETC mtDNA deletion process is active, and which becomes activated after cessation of $\mathrm{O}_{2}^{-}$stress to eradicate ETC mtDNA deprived genomes.

\section{Regulatory Scope of the Genetically Programmed Adaptation}

Our data show that if a temporary oxidative stress is induced in a large number of mitochondria, mitochondrial fragmentation and subsequent ETC mtDNA deletions provide a means to lessen overall cellular $\mathrm{O}_{2}^{-}$stress without reducing mitochondrial 
volume. In contrast, to achieve a reduction in overall $\mathrm{O}_{2}^{-}$stress by canonical mitophagy alone, the mitochondrial volume would have to be substantially reduced. Because mitochondria are deeply integrated into cellular physiology and are needed for a range of cellular functions, some of which are essential to cell survival, the mitophagic strategy is arguably a less favorable option. In this context, natural selection would therefore have ample room to accommodate the establishment of a targeted mtDNA deletion mechanism.

Canonical mitophagy has been suggested to be responsible for the removal of deleterious ETC gene mutations causing local intramitochondrial oxidative stress (Bess et al., 2012; Lemasters, 2005; Palikaras \& Tavernarakis, 2014; Sedlackova \& Korolchuk, 2019). As targeted ETC mtDNA deletion appears to be a much more efficient way to achieve such local damage control, both in terms of response time and cellular cost, it is plausible that this mechanism may also operate independently of a fragmented mitochondrial morphology.

\section{Why is there No Regulatory Break Preventing Mitochondrial Impairment?}

It is quite intriguing that the mtDNA deletion mechanism remains active even if all mtDNA genomes in a cell have lost almost their entire $77 \mathrm{~kb}$ DNA content, and one may ask why the yeast has not evolved any mechanism to prevent this apparently maladaptive behavior. Wild yeast rarely proliferates longer than a few generations before nutrient depletion forces cells into meiosis and the production of resting spores (De Chiara et al., 2020). Our results therefore imply that even if cells are exposed to oxidative stress for their entire asexual growth period, they would still be in a heteroplasmic state when the next growth cycle starts. Thus, there is unlikely to have been a sufficient evolutionary reason to counteract the observed loss of 
restorative capacity. The same line of reasoning applies to mammalian systems as the physiological consequences of chronic $\mathrm{O}_{2}^{-}$stress associated with high age have barely been exposed to natural selection in the wild (Medawar, 1952).

\section{Do the Results Extend to Humans?}

As targeted ETC mtDNA deletion, in full accord with the Co-location for Redox Regulation (CoRR) hypothesis (Allen, 2017; Johnston \& Williams, 2016), appears to enhance the capacity for localized and optimized maintenance of the delicate balance between energy production and $\mathrm{O}_{2}^{-}$stress, it is unlikely to be a regulatory mechanism that has been monopolized by budding yeast.

Oxidative stress markers associate with age and all-cause mortality (Schöttker et al., 2015). Ageing is associated with high circulating levels of proinflammatory markers (Ferrucci \& Fabbri, 2018), proinflammatory cytokines induce ROS production in several cell types (Yang et al., 2007), and oxidant overproduction is a candidate mechanism in neuronal damage and loss via neuroinflammation (Picca et al., 2020), a common denominator of neurodegenerative diseases (van Horssen, van Schaik, \& Witte, 2019). Thus, assuming that the level of chronic $\mathrm{O}_{2}^{-}$ stress sensed by mitochondria increases with age, our results suggest an explanation for why the levels of mtDNA deletions increase with age in tissues such as the brain, retina, skeletal muscle, sperm, ovaries, hepatocytes and heart, and for why mtDNA deletions appear to contribute to the occurrence of age-related OXPHOS dysfunction (Kauppila, Kauppila, \& Larsson, 2017). Moreover, most mtDNA deletions reported in humans are affecting the major arc of mtDNA, containing COX1, COX2, COX3, ATPs 6 and ATPs8, and the majority of the large deletions are flanked by homologous or near-homologous repeats. This corresponds 
very well with the $\mathrm{O}_{2}^{-}$-induced deletions we observe in the early phase of paraquat adaptation in yeast (Figure 3D; Figure S4), as well as with our conjecture that the deletions may be driven by a MMEJ mechanism.

Although the dominant theory is that mtDNA deletions arise spontaneously at microhomologous sites and undergo clonal expansion (Nido et al., 2018), the exact mechanisms remain elusive. Our results provide an explanation for why certain mtDNA deletions become common under oxidative stress, and they also suggest that what might appear as clonal expansion, may in some cases be due to targeted mtDNA deletion in many mitochondrial genomes and subsequent replication of these genomes while oxidative stress prevails.

\section{ACKNOWLEDGMENTS}

The authors acknowledge Illumina sequencing technical support from the Science for Life Laboratory (SciLife), the National Genomics Infrastructure, NGI and National Bioinformatics Infrastructure in Sweden and PacBio sequencing technical support from the Norwegian Sequencing Centre. We acknowledge the Centre for Cellular Imaging at the University of Gothenburg and the National Microscopy Infrastructure (VR-RFI 2016-00968) for assistance with the confocal microscopy. We thank Johan Hallin and Lars-Göran Ottosson for help and advice with strain construction and design of adaptation experiment, Olga Kourtchenko for help with designing nitrogenlimited environments, and Tom Kirkwood for instrumental comments to an earlier version of this paper. Funding: This work was supported by the Swedish Research Council (2014-6547, 2014-4605, 2015-05427, 2018-03638 and 2018-03453), the Research Council of Norway (178901/V30 and 222364/F20), Knut and Alice Wallenberg Foundation, Agence Nationale de la Recherche (ANR-11-LABX-0028- 
01, ANR-13-BSV6-0006-01, ANR-15-IDEX-01, ANR-16-CE12-0019 and ANR-18CE12-0004) and Cancerfonden (2017-778).

\section{AUTHOR CONTRIBUTIONS}

SWO and JW conceived and coordinated the study with help from MM, ABG and GL. SWO, ABG, JW and SS designed evolution experiments. SS, KP, CG and PG performed, analyzed and visualized evolution experiments. SWO, JW and GL designed DNA sequencing experiments. SS, JL, JXY and CG performed, analyzed and visualized DNA sequencing experiments. SWO, JW and SS designed qPCR experiments. SS and EDH performed, analyzed and visualized qPCR experiments. SS, LL and JLH designed electron microscopy experiments. SS, LL performed, analyzed and visualized microscopy experiments. SS, JL, JW and GL designed aneuploidy experiments. JL and SS constructed strains and performed aneuploidy experiments. SS, MZ, ABG, SWO and JW designed simulations. SS, ABG, MZ and TÄ performed, analyzed and visualized simulations. JW, SS and KP designed schematic figures. SS, SWO and JW wrote the paper, with input from all other authors.

\section{DECLARATION OF INTERESTS}

The authors declare no competing interests.

\section{FIGURE LEGENDS}

\section{Figure 1. Swift Initial Adaptation to $\mathrm{O}_{2}^{-}$Generated by Paraquat}

(A) Model of paraquat induced $\mathrm{O}_{2}^{-}$generation in yeast mitochondria. ETC complex III and mitochondrial NADPH dehydrogenases donate electrons to paraquat, which passes these on to $\mathrm{O}_{2}$ (Castello et al., 2007; Cochemé \& Murphy, 2008). This mode 
of $\mathrm{O}_{2}^{-}$generation resembles the natural process, where electrons leak from the mitochondrial ETC directly to $\mathrm{O}_{2}$, primarily from ETC complex I and III (Fang \& Beattie, 2003; Turrens, 1997). (B) RNA expression (relative no stress at $t=0, \log _{2}$ ) of $C C P 1, S O D 1$ and $S O D 2$, during the first, second and third growth cycle in presence and absence of $\mathrm{O}_{2}^{-}$stress. Error bars: S.E.M. (n=3) (C) Adaptation to $\mathrm{O}_{2}^{-}$stress (paraquat) and seven other challenges. Mean of 96 adapting populations (each at $n=6$ ) is shown. Shade: S.E.M. (D) Adaptation to $\mathrm{O}_{2}^{-}$stress of 96 experimental and 1152 simulated populations. We based simulations on empirical estimates of rates and effect sizes of point mutations, chromosome duplications or both. Shade: S.D. (E) Adaptation to $\mathrm{O}_{2}^{-}$stress after relaxing selection post $6,10,19,24,33$ and 242 generations (panels) of $\mathrm{O}_{2}^{-}$adaptation. Lines: 96 populations (each at $n=5$ ). (F) Doubling time $(h)$ difference between $\mathrm{O}_{2}^{-}$adapted and founder cells in the absence of stress, after $6,10,19,24,33$ and 242 cell generations of $\mathrm{O}_{2}^{-}$adaptation. Right $y$-axis: Converting doubling time difference into selection coefficient, s. Means of 96 populations (each at $n=5$ ) are shown. Error bars: S.E.M. See also Figure S1-S2.

\section{Figure 2. Mitochondrial Fragmentation During $\mathrm{O}_{2}^{-}$Adaptation}

(A) EM microscopy of cells before (Oh) and during early (7h) $\mathrm{O}_{2}^{-}$stress. Red arrowheads: Representative mitochondria. Left diagram: Mitochondria per cell (count). Right diagram: Imaged cell area occupied by mitochondria (\%), used as proxy for mitochondrial volume. Error bars: S.E.M. ( $n=100$ cells). (B) Confocal microscopy of cells with a Cox4-GFP mitochondrial tag, before (0h) and during early (7h) $\mathrm{O}_{2}^{-}$stress, after 1 growth cycle $(72 \mathrm{~h})+7 \mathrm{~h}$ of $\mathrm{O}_{2}^{-}$stress adaptation and $7 \mathrm{~h}$ after release from 2 cycles $(144 \mathrm{~h})$ of $\mathrm{O}_{2}^{-}$adaptation. Color: $z$-dimension (yellow=front, black=back). See also Figure S3. 


\section{Figure 3. Early $\mathrm{O}_{2}^{-}$Adaptation Targets the ETC}

(A) Adaptation to $\mathrm{O}_{2}^{-}$stress coincides with loss of respiratory (glycerol) growth. Shade: S.E.M. across 96 adapting populations (each at $n=3$ ). Broken line: no growth (cell doubling time>24h). (B) Recovery of respiratory (glycerol) growth (right $y$-axis, purple line) and loss of $\mathrm{O}_{2}^{-}$adaptation (left $y$-axis, green line) in cells released from 0 and 6 cell generations of $\mathrm{O}_{2}^{-}$stress. Shade: S.E.M. of 5 populations (as in 2C), each at $n=5$. (C) mtDNA copy number change (left $y$-axis, red line, median coverage relative to the haploid nuclear genome) during $\mathrm{O}_{2}^{-}$adaptation (right $\mathrm{y}$-axis, green line, $n=6$ ) for 5 populations (panels). Shade: S.E.M. (D) Diagrams: mtDNA copy number change (left $y$-axis, purple line) of individual mtDNA genes during $\mathrm{O}_{2}^{-}$adaptation (right $y$-axis, green line) in population A7. Error bars: S.E.M. $(n=3)$. Circle: mtDNA $(77 \mathrm{kB})$ before $\mathrm{O}_{2}^{-}$stress adaptation. Genes, origins of replication and position (kb) are indicated. Coloured fields: mtDNA deletions with concerted copy number change. (E) Recovery of mtDNA copy number ( $y$-axis, median coverage in $1 \mathrm{~kb}$ windows relative euploid nuclear genome) after release from 6 generations of $\mathrm{O}_{2}^{-}$ stress (dot colour: $G_{r}$ ). Red bars: mtDNA deletion. $(F)$ Recovery of the copy number of mtDNA deletions (right $y$-axis, red line; from Figure 2E) after release from 6 generations of $\mathrm{O}_{2}^{-}$stress coincides with loss of $\mathrm{O}_{2}^{-}$adaptation (left $y$-axis, green line). See also Figure S4.

\section{Figure 4. Chromosome Duplications Explain the Second $\mathrm{O}_{2}^{-}$Adaptation Phase}

(A) Chromosome II, III and V duplications are common after 242 generations $\left(t_{50}\right)$ of $\mathrm{O}_{2}^{-}$stress. Colour: Chromosome copy number $\left(\log _{2}\right.$ median coverage relative to haploid nuclear genome. (B) Chromosome II and III duplications reduce the cell 
doubling time during $\mathrm{O}_{2}^{-}$stress. We backcrossed $(\mathrm{x} 3)$ cells adapted to 242 generations $\left(t_{50}\right)$ of $\mathrm{O}_{2}^{-}$stress to founder cells over consecutive meioses and compared the $\mathrm{O}_{2}^{-}$stress growth of 2-3 segregants with and without duplicated chromosome. $y$-axis: Doubling time relative founder $\left(\log _{2}\right)$. Error bars: S.E.M. $(n=6)$. See also Figure S5.

Figure 5. Chronic $\mathrm{O}_{2}^{-}$Stress Causes Irreversible Mitochondrial Impairment by Sustained mtDNA Deletion

(A) Cells adapting to $\mathrm{O}_{2}^{-}$stress (panels; generations of adaptation) lose the capacity to recover respiratory (glycerol) growth (right $y$-axis, purple line) when released from $\mathrm{O}_{2}^{-}$stress and the loss coincides with fixation of $\mathrm{O}_{2}^{-}$adaptation (left $y$-axis, green line). Shade: S.E.M. of 5 populations (as in 2 ), each at $n=5$. (B) Cell populations adapted to chronic $\mathrm{O}_{2}^{-}$stress $\left(t_{50}\right)$ retain all $\left(\rho^{+}\right)$, large $\left(6-30 \mathrm{~kb} ; \rho^{-}\right)$or small $(<2 \mathrm{~kb}$, $\rho^{--}$) mtDNA segments. Panels: Representative examples. $y$-axis: mtDNA copy number (median coverage in $1 \mathrm{~kb}$ windows relative to haploid nuclear genome). Gene positions are indicated. (C) Number of $\rho^{-}$populations adapted to chronic $\mathrm{O}_{2}^{-}$stress $\left(t_{50}\right)$ in which the specified mtDNA gene was lost. (D) $\rho^{--}$populations became less fit than $\rho^{-}$during the second phase of $\mathrm{O}_{2}^{-}$adaptation. See also Figure S6.

Figure 6. Schematic Interpretation of $\mathbf{O}_{2}^{-}$Stress Adaptation

Paraquat generates mitochondrial $\mathrm{O}_{2}^{-}$stress through redox cycling (Figure 1A). Cells register the stress, induce mitochondrial fragmentation (Figures $2 \mathrm{~B}$ and $\mathrm{C}$; Figure S3A) and activate a regulatory system deleting mtDNA encoding ETC components while otherwise retaining mtDNA homeostasis (Figures 3C and 3D; Figure S4). This 
causes a substantial reduction in mitochondrial $\mathrm{O}_{2}^{-}$production, which leads to the observed swift adaptation (Figure1B; Figure S2A) and swift loss of respiratory capacity (Figure 3A). However, as paraquat is redox cycled also by nuclear encoded mitochondrial NADPH dehydrogenases (Cochemé \& Murphy, 2008) and cytoplasmic Yno1 (Rinnerthaler et al., 2012), the deletions of mtDNA encoding ETC components fail to completely remove the $\mathrm{O}_{2}^{-}$stress. This causes continued operation of the regulatory system and thus continued removal of mtDNA segments. Nevertheless, as long as the cells are still in a heteroplasmic state (also containing intact mtDNA genomes), removal of the $\mathrm{O}_{2}^{-}$stress causes removal of non-intact mtDNA genomes (Figures 3E and 3F) and restitution of the tubular mitochondrial morphology (Fig 2B) and respiratory capacity (Figure 3B). If cells are exposed to paraquat until there are no intact mtDNA genomes left (Figures 5C and 5D; Figure S6A), restitution of the respiratory capacity becomes impossible (Figure 5B).

\section{STAR* METHODS}

\section{Yeast Cells Used}

Founder strain: We used a single, haploid clone of the yeast strain YPS128 (MATa ura3::NatMX-barcode ho::HYGMX) as founder genotype. YPS128 is a wild, oak isolate with a North American genome composition (Liti et al., 2009). In contrast to common domesticated lab-strains, YPS128 has growth typical of the species (Warringer et al., 2011), excellent respiratory growth (De Chiara et al., 2020), and no confounding transposon insertion (Gaisne, Bécam, Verdière, \& Herbert, 1999) in the transcription factor Hap1, which regulates nuclear and mitochondrial genes in response to oxygen. Aneuploidic strains: We reconstructed individuals with single chromosome duplications (II, III, IV, V, VI, VIII, IX, X, XI, XII, XIII, XIV, XV and XVI) 
by two methods. Duplications of I and VII could not be obtained by either methods, despite repeated tries and may be lethal in YPS128. First, to construct duplications of chromosome II, III, V, X and XVI, we repeatedly (3x) backcrossed clones from endpoint $\left(t_{50}\right)$ populations carrying chromosome duplications to founder clones of the opposite mating type (MATa ho::HYGMX). Each backcross was performed on YPD (Yeast Peptone Dextrose) medium using haploids verified by qPCR to retain the chromosome duplication, diploid hybrids were selected after three days of growth on solid minimum media (0.675\% Yeast Nitrogen Base (CYN2210, ForMedium), 2\% (w/v) D-Glucose, $\mathrm{pH}=6-6.5(\mathrm{NaOH}), 2.5 \%$ agar) medium and sporulated overnight on solid $1 \%$ potassium acetate sporulation medium to generate recombined haploids. These were genotyped at the URA and ho locus and ura- MATa haploids were passed on to the next round of backcrossing. After three rounds of backcrossing, we selected ura- MATa haploids with ( $n=2$ clones) and without ( $n=2$ clones) the chromosome duplication of interest and estimated their respective fitness (see below) at high biological replication $(n=6)$ and in a completely randomized design on the media of interest. We estimated the fitness (doubling time, $D$ ) effect of the chromosome duplication by comparing (by subtraction) the doubling time of haploids with and without chromosome duplication(s). We assumed that additional mutations affecting doubling time in the adapted clones segregate independently of the chromosome duplication. We obtained parts of the empirical data underlying the simulations in Figure 1D and the empirical data in Figure 4B and Figure S4C using these constructs. Second, we reconstructed the chromosome duplications IV, VI, VIII, IX, XI, XII, XIII, XIV and XV as in Zebrowski et al., 2008 (Zebrowski \& Kaback, 2008). We genetically modified the founder clone genotype to match the his3 $\Delta$ (complete deletion by transformation with pSH47) and can1::STE2pr-HIS3 genotype 
of the aneuploidic construct, as described in Zebrowski et al., 2008 (Zebrowski \& Kaback, 2008), and used these as marker neutral controls. We obtained parts of the empirical data underlying the simulations in Figure 1D using these constructs.

Gene deletion strains: To estimate gene loss-of-function mutation effect sizes underlying simulations shown in Figure 1D, we used the haploid BY4741 single gene

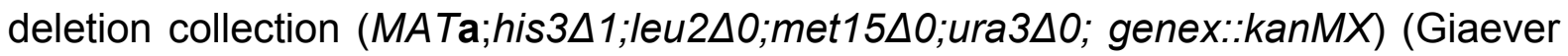
et al., 2002) for growth in absence and presence of each stressor. Collection size: $n=4580$, each cultivated at $n=6$. We also generated a true $\rho^{0}$ strain, lacking all mtDNA, by deleting the sole yeast mitochondrial DNA polymerase Mip1, from start to stop codon. Genotype: YPS128 MATa ura3::NATMX-barcode ho::HYGMX $\Delta$ mip1::KanMX. We used mip1 $\Delta$ as a mtDNA negative control in Figure S6B. To construct Cox4-EGFP fusion as a reporter for mitochondrial morphology, founder cells were transformed with PCR fragments of EGFP amplified from pYM27, with flanking regions homologous to COX4. Downstream of COX4 we inserted kanMX as selection marker during transformation. Cox4 localises to the mitochondrial inner membrane (J. Zhu et al., 2019). We used Cox4-EGFP as a mitochondrial signal in Figure 2B and Figure S3A. All primers are reported in Supplementary Data S19.

\section{Cell Cultivation Media Used}

Except where otherwise stated, yeast strains were cultivated on a Synthetic Complete medium (SC; hereafter: "Background medium") composed of $0.14 \%$ Yeast Nitrogen Base (CYN2210, ForMedium), $0.50 \% \quad \mathrm{NH}_{4} \mathrm{SO}_{4}, \quad 0.077 \%$ Complete Supplement Mixture (CSM; DCS0019, ForMedium), 2.0\% (w/w) glucose, $\mathrm{pH}$ set to 5.80 with $1.0 \%(\mathrm{w} / \mathrm{v})$ succinic acid and $0.6 \%(\mathrm{w} / \mathrm{v}) \mathrm{NaOH}$. For solid medium cultivations, $2.0 \%(\mathrm{w} / \mathrm{v})$ agar was added. For pre-cultures to glycine, isoleucine, 
citrulline and tryptophan selection environments, we modified the background medium to avoid nitrogen storing and later confounding growth on the stored nitrogen (Gutiérrez, Sancho, Beltran, Guillamon, \& Warringer, 2016) we replaced CSM by $20 \mathrm{mg} / \mathrm{L}$ uracil, which is not used or stored as a nitrogen source, and we drastically reduced the $\mathrm{NH}_{4} \mathrm{SO}_{4}$ to a concentration (30 mg N/L) limiting the cell growth yield. Selection environments represented simple modifications to the background medium: $+0.8 \mu \mathrm{g} / \mathrm{mL}$ rapamycin, $+400 \mu \mathrm{g} / \mathrm{mL}$ paraquat (methylviologen; N,N-dimethyl-4-4'-bipiridinium dichloride) (Cochemé \& Murphy, 2008), +3 mM arsenic ([As III]; $\mathrm{NaAs}_{2} \mathrm{O}_{3}$ ), $+62.5 \mathrm{mg} / \mathrm{L}$ citric acid. For the four nitrogen selection experiments environments, we replaced $\mathrm{NH}_{4} \mathrm{SO}_{4}$ in the nitrogen background medium with $30 \mathrm{mg} \mathrm{N} / \mathrm{L}$ of L-glycine, L-isoleucine, L-citrulline or L-tryptophan, together with $20 \mathrm{mg} / \mathrm{L}$ uracil. For the respiratory growth experiments, we replaced $2 \%$ glucose with $2 \%$ glycerol as the sole carbon and energy source. Because of chemical batch variations, the stress imposed by a particular challenge may vary somewhat across experiments. We cast all solid plates $10-15$ hours prior to use, on a level surface, by pouring $50 \mathrm{~mL}$ of selection medium in the same upper right corner of each plate. We removed excess liquid by drying plates in a laminar airflow in a sterile environment. All yeast populations were stored at $-80^{\circ} \mathrm{C}$ in $20 \%$ glycerol and cultivated at $30^{\circ} \mathrm{C}$. Populations were subsampled and transferred to and from evolution plates, storage plates and experimental plates using robotics (ROTOR HDA, Singer Instruments), at the indicated transfer format.

\section{Experimental Evolution of Cells}

We single streaked and expanded a haploid founder clone to moderate colony size ( $\sim 2$ million cells). We subsampled the colony randomly ( $\sim 0000$ cells), expanded it 
until stationary phase ( 2 million cells; $36 \mathrm{~h})$ in $5 \mathrm{~mL}$ of background medium, poured the culture on top of a solid plate (background medium; cast in PlusPlates, Singer Instruments, UK) and expanded the population again until stationary phase $(72 \mathrm{~h})$, creating a lawn of founder cells. A subsample of lawn cells was stored in $-80^{\circ} \mathrm{C}$, hereafter referred to as "lawn". We subsampled the lawn and transferred 1152 subsamples ( 150.000 cells) to each of eight fresh solid plates, using robotics (ROTOR HDA, Singer Instruments) and 384 short pin pads. These served as precultures $\left(t_{-1}\right)$ to the first selection cycle. We propagated pre-cultures on background, or nitrogen background, medium. We expanded pre-cultured populations into stationary phase ( 2 million cells; $72 \mathrm{~h})$. A subsample of each stationary phase preculture was then stored at $-80^{\circ} \mathrm{C}$. The stationary phase pre-cultures were randomly subsampled ( $\sim 50.000$ cells) by robotics and 1536 short pin pads to create individual founder populations for each position; we refer to these as $t_{0}$ founder populations throughout the paper. We designed the preparation procedure to minimize genetic, epigenetic and plastic (environmental) variation in cellular states, between and within populations and plates. We note that none of these parameters will be exactly zero because of: a) the need for population expansions and cell divisions b) population genetic and environmental structure in colonies. We initiated adaptation experiments by transferring (384 short pin pads; robotics) 1152 subsampled ( 50.000 cells) founder populations to selection plates containing the stresses in Table S1. We propagated populations for $72 \mathrm{~h}$ until stationary phase before repeating the subsampling. We cycled all populations through 50 consecutive rounds of population expansion and subsampling to generate samples $t_{1}$ to $t_{50}$. All cycles were initiated and terminated identically. While the design minimized environmental and epigenetic variation over time and space, we note that these parameters will not be exactly zero 
because a) the physical boundaries of plates and slight variations in medium solidification and water evaporation within and between plates creates a somewhat structured environment, b) the time spent in stationary phase before transfer increases as populations adapt, and c) for each cultivation cycle, any inherited epigenetic effects from the pre-cultivation is systematically shifted backwards in time. To account for systematic variations, we randomized samples as far as logistically possible and normalized all data post-experimentally.

\section{Cell Cultivation for Estimation of Cell Growth}

Adapting populations: We subsampled (1536 short pin pads) all 9216 adapting cell populations at the end ( $72 \mathrm{~h})$ of each batch-cycle. We tracked their population size expansion during a subsequent batch cultivation $(n=1$, no randomization of samples). The separation of adaptation and cell growth phenotyping allowed the interleaving (384 short pin pads) of 384 non-evolved fixed controls (founder genotypes) among evolving populations, and the use of these as spatial controls to reduce the effect of systematic variations within and between plates. This shifted the time vector of measured relative evolved states, i.e. true $t_{0}$ zeros samples not having passed through any preceding stress cultivation, are missing. We account for this by assuming that true $t_{0}$ samples would have identical properties to $t_{1}$ samples, while acknowledging that this disregards any adaptation occurring in the 1st batch cycle ( 2-2.5 cell doublings in paraquat exposed populations). We report thie data in Supplemental Information Data S1 and visualize them in Figure S2A.

Chronological record populations: We systematically subsampled ( $~ 50.000$ cells per population) the same 96 random populations at the end of batch cycles $0,1,2,3,4$, $5,7,9,12,15,20,25,30,35,40,45$ and 50 in each selection regime. We expanded 
subsampled populations until stationary phase $(72 \mathrm{~h})$ in liquid, selection medium micro-cultures $(100 \mu \mathrm{L}$ ), added $100 \mu \mathrm{L}$ of glycerol (final concentration: $20 \%(\mathrm{w} / \mathrm{w})$ ) and stored the 768 adapting populations to create a frozen chronological record at ($\left.80^{\circ} \mathrm{C}\right)$. We thawed the frozen chronological record at room temperature, resuspended, subsampled ( 50.000 cells), transferred subsamples to solid background or nitrogen background medium, and propagated populations until stationary phase $(72 \mathrm{~h})$ to create no stress pre-cultures. We subsampled pre-cultures, transferred subsamples to solid medium selection plates in a well replicated $(n=6)$ design with near complete randomization of replicates and adaptation stages, within and between plates and instruments. Randomization was achieved using the randint function in the python package NumPy (version 1.15.4). We interleaved 384 spatial controls (as for the 9216 adapting populations; frozen in absence of the stressor) among evolving populations. Note that the cultivation for freezing systematically shifted the time vector of measured relative evolved states, as for the 9216 populations: i.e. measurements for $t_{1}$ samples are extracted during their second round of stress cultivation and true $t_{0}$ zeros samples, not having passed through a preceding stress cultivation, are missing. We handled this postexperimentally as for the 9216 adapting populations. We also note that we cannot exclude that the pre-cultivation in absence of stress may affect the genetic and/or epigenetic states of populations somewhat. We report these data in Supplemental Information Data S2 and S3, and visualize them in Figures 1C, 1D, 3A, 3D and 5D, and Figures S4, S5B, S5C and S6D.

Release-from-selection populations: To test whether adapted phenotypes were reversible or fixated, we tracked the capacity of frozen chronological populations to retain adaptive states over the first $1-11$ batch cycles after release from a selection 
pressure. First, we thawed the $t_{1}, t_{2}, t_{3}, t_{4}, t_{5}, t_{7}$ and $t_{50}$ frozen chronological states of the 96 paraquat adapted populations, re-suspended each thawed population state, subsampled these robotically and deposited $\sim 50.000$ cells of each on no stress solid medium plates. We evolved populations over ten ( 84 generations) consecutive cycles of population expansion and subsampling on no stress plates, i.e. under relaxation of the paraquat selection. At the end $(72 \mathrm{~h})$ of each evolution cycle, evolving populations were preserved in glycerol at $-80^{\circ} \mathrm{C}$ (as above), creating a new, standardized chronological record of samples frozen after different time periods of release from the original selection. This frozen chronological record was again thawed, re-suspended, subsampled and transferred to 1536 pre-cultivation plates (no stress) with founder populations handled identically. All samples were randomized (as above) across plates, positions and scanning instruments. At stationary phase $(72 \mathrm{~h})$, pre-cultures were subsampled four times $(n=4)$ and transferred to fresh paraquat plates, with randint randomization of replicates (as above), and measured the population size expansion of these populations. Again, while the pre-cultivation step is required for standardization, we note that it systematically shifts the time vector of measured relative evolved (relaxed selection) states. Thus, the absence of true $t_{0 \text {-relaxation }}$ samples, not having passed through any cycles of relaxed selection, we conservatively assumed that these would have the same properties as the measured $t_{1 \text {-relaxation }}$ samples, i.e. no loss of adapted phenotype during the first cycle of relaxation. We report these data in Supplemental Information Data S4 and S5 and visualize them in Figure 1E and 1F. To compare the swift loss of adapted phenotypes in paraquat populations released from selection to that of populations adapted to other environments characterized by fast adaptation, we repeated the selection relaxation experiment on the 96 populations adapting to 
paraquat, arsenic and glycine, with the selection independently repeated three times for each population. For each environment, we selected a single time point in the chronological record, corresponding to the 96 populations having reached $70-90 \%$ of the endpoint adaptation, thawed and subsampled these, expanded them under relaxed selection for 10 evolution cycles of population expansion and subsampling, created a new frozen chronological record; this was revived, pre-cultivated and cultivated in presence of the original stress ( $n=5$, randomization). All procedures were identical to the first relaxation experiment. Relaxation of selection for glycineadapted populations was performed in nitrogen-limited background medium. We report the data in Supplemental Information Data S6 and visualize them in Figures S2B and S2C. Finally, we repeated the selection relaxation experiment a third time, for the five sequenced paraquat-adapting populations (A7, A8, B12, B5 and B8). Procedures were as above. We repeated the selection relaxation three times for each frozen chronological state of each population and we tracked both paraquat tolerance and respiratory (glycerol) growth both at $n=5$ (randomization). We report these data in Supplemental Information Data S7 and visualize them in Figures 3B and $5 \mathrm{~A}$.

Deletion collection: To generate the effect size estimates of loss-of-function mutations that form part of the empirical basis for the modelling in Figure 1D, we thawed the frozen deletion collection at room temperature, re-suspended, subsampled and transferred subsamples ( 50.000 cells) to solid background medium. At stationary phase $(72 \mathrm{~h})$, pre-cultures were subsampled six times $(n=6)$ and transferred to experimental plates. Three of these subsamples were distributed across each of the two experimental plates, in fixed positions, for a total of $n=6$ 
replicates. We report these data in Supplemental Information Data S8, and they underlie the simulation results in Figure $1 \mathrm{D}$.

Aneuploidies: To generate the effect size estimates of aneuploidies that form part of the empirical basis for the simulations in Figure 1D, we thawed the frozen aneuploidic strains at room temperature, re-suspended, subsampled and transferred subsamples ( 50.000 cells) to solid background medium. At stationary phase $(72 \mathrm{~h})$, pre-cultures were subsampled nine times and subsamples were transferred to experimental plates. Three of the subsamples were distributed across each of the three experimental plates, in fixed positions, for a total of $n=9$ replicates. We report these data in Supplemental Information Data S9, they underlie the simulations in Figure 1D and they are visualized in Figure 4B.

\section{Counting Cells in Growing Populations}

We tracked population size expansion for all cultures using the Scan-o-matic system (Zackrisson et al., 2016) version 1.5.7 (https://github.com/Scan-oMatic/scanomatic.git). We deposited 1152 experimental populations (1536 short pin pads) on each experimental plate. To account for spatial variation across plates, without introducing bias from using the experimental values for normalization, we introduced (384 short pin pads) 384 controls (founder genotypes) in the 384 interleaved empty positions on each experimental plate. We subsampled controls from a separate 384 pre-culture array, pre-cultivated in parallel to experimental cultures. The initial population size is on average 1.55 fold larger for spatial controls and their history prior to the pre-culture differs from that of experiments; thus, they were used to capture spatial variation, and not as true $t_{0}$ samples. Plates were maintained undisturbed and without lids for the duration of the experiment $(72 \mathrm{~h})$ in 
high-quality desktop scanners (Epson Perfection V800 PHOTO scanners, Epson Corporation, UK) standing inside dark, temperature $\left(30.0^{\circ} \mathrm{C}\right)$ and moisture controlled thermostatic cabinets with intense air circulation. Scanners were connected via USB to standard desktop computer. Scanner power supplies were separately controlled by power managers that instantaneously shut down the scanner after scans, avoiding light stress. Images, capturing four plates per image, were acquired using SANE (Scanner Access Now Easy) and transmissive scanning at $600 \mathrm{dpi}$. Plates were fixed in place by custom-made acrylic glass fixtures. Orientation markers contained on each fixture ensured pixel-exact recognition of plate positioning. Each fixture was calibrated by scanner using a calibration model that provided positions for each feature of that fixture, relative to its orientation markers. Pixel intensities were normalized and standardized across instruments and time using transmissive scale calibration targets (LaserSoft IT8 Calibration Target, LaserSoft Imaging, Germany). This accounts for changes in light intensity due to lamp or sensor aging as well as other changes in the light environment, across the whole pixel intensity spectrum. Each image stack was processed in a two-pass analysis. The first-pass was performed during image acquisition to set up the information needed for population size estimations. Positions in each image were matched to the fixed calibration model using the fixture orientation markers, allowing detection and annotation of plates and transmissive scale calibration strips. In the second-pass analysis, images were segmented to identify plate and transmissive scale calibration strip positions. The calibration strips were trimmed and the pixel intensities compared to the manufacturer's supplied values, such that normalized pixel values remained independent of shifts in light properties of e.g. the light source or detector. Colonies were detected using a virtual grid across each plate based on pinning 
format, and the grid was adjusted such that intersections matched the center of the features detected. At every intersection, each colony and the surrounding area were segmented to determine the local background and pixel intensities. Differences in pixel intensity were converted to population size estimates by calibration to a preestablished, independent calibration function, obtained using cell number estimates from both spectrometer and flow cytometer measurements. Based on these cell counts, we obtained population size growth curves. Each series of population size measures were smoothed in a two-step procedure to account for random variation. First, a median filter identified and removed local spikes in each curve. Second, a Gaussian filter reduced the influence of remaining local noise.

\section{Cell Growth and Evolution Parameters}

Estimating cell doubling time and adaptation: We identified the steepest slope in each growth curve by local regression over five consecutive time points and converted slopes into population size doubling times. For quality control, the residuals of the regression model were used to determine goodness-of-fit and to flag growth curves suspected to be of poor quality. We manually inspected all flagged growth curves, discarding approximately $0.3 \%$ as erroneous. We extracted the population size doubling time, $D(h)$, for all retained populations and log transformed it as $\log _{2}(D)$. To minimize systematic errors caused by structured environmental variation within and between plates, we used the fixed spatial controls (founders) introduced at every fourth position. First, controls with extreme $\log _{2}(D)$ were removed and the remaining control positions were used to interpolate a normalization surface across all 1536 positions on the plate; i.e. we estimated what $\log _{2}(D)$ value a control would have in each position. Second, the interpolated surface across 1536 positions 
was smoothed, first with a kernel filter to exclude deviant positions, and then with a Gaussian smoothing $(\sigma=1.5)$ to soften the contours of the control landscape. Third, for each of the 1536 positions, we estimated the expected control value, $\log _{2}(D)$, by calculating the distance-weighted (Gauss distribution, $\sigma=1.5$ ) local mean of the smoothed control landscape. Fourth, we extracted the normalized, relative population size doubling time for each experiment, $D_{\text {norm }}$, by subtracting the control value for that position as $D_{\text {norm }}=\log _{2}\left(D_{\text {experiment }}\right)-\log _{2}\left(D_{\text {control }}\right)$. $D_{\text {norm }}$ is reported in Supplemental Information Data S1, S3, S4, S6, S7, S8, and S9, as left y-axis data in Figures $3 \mathrm{~F}$ and $4 \mathrm{~B}$ and Figure $\mathrm{S} 2 \mathrm{~A}$, and as right $y$-axis data in Figures $3 \mathrm{~A}, 3 \mathrm{~B}$ and 5A. For adapting populations the spatial control samples differ slightly in initial population size, cultivation and storage history from other samples, creating a small but systematic error. We removed this error by estimating the adaptation, $A$, achieved up to each batch cycle as: $D_{\text {norm,t }}-D_{\text {norm,o. }} A$ is reported in Supplemental Information Data S2 and visualized as left $y$-axis data in Figures 1C, 1D, 1E, 3A, 3B, $3 C, 3 D, 5 A, 5 D$ and Figures S2B, S4 and S6D, and as right $y$-axis data in Figures S5B and S5C. In Figure S6C, we converted $D_{\text {norm }}$ back to a normalized absolute doubling time (h), $A b s_{n o r m}$, as $A b s_{n o r m}=2^{D n o r m} D_{\text {control, grand. }} D_{\text {control, grand }}$ is the grand mean of all control doubling times run in the particular experimental series, removing plate and batch bias and allowing direct comparison across experiments. In Figure $1 \mathrm{~F}$ and Figure $\mathrm{S} 2 \mathrm{C}$, we show the difference between the normalized absolute doubling times $(h)$ of adapted and adapted populations in no stress.

Counting cell generations: We estimated the cell generations completed in each batch cycle, for each population, as the number of population size doublings from batch cycle start to end, i.e. to the time of subsampling for transfer the next batch cycle. Assuming no cell death, the number of population size doublings correspond 
to the mean number of consecutive cell divisions and thus the mean number of cell generations. We estimated cell generations for missing batch cycles by linear interpolation between values estimated for the immediately adjacent measured batch cycles. Summing over all batch cycles up to a given time point, we obtained measures of the total number of cell generations under each selection pressure, $G_{s}$, and the total number of cell generations after release from each selection pressure, $G_{r}$. We show the mean $G_{s}$ and the mean $G_{r}$ across all replicate measures of all evolving replicate populations at that stage (cycle) of evolution. We note that while $G_{s}$ and $G_{r}$ estimate the number of generations to the end of each batch cycle, the doubling time estimate $D$ is extracted from the exponential phase of the same batch cycle; this leads to a very slight mismatch, which we cannot account for. We report $G_{s}$ in Supplemental Information Data S1-S4 and S7, and $G_{r}$ in Supplemental Information Data S4 and S6.

Estimating the maximum possible adaptation: We postulated that the cell doubling time of YPS128 in absence of stress represents an absolute, lower boundary for the doubling time achievable by YPS128 in any selection environment. To retain the spatial normalization, while working at the level of absolute doubling time (in hours), we converted the $D_{\text {norm }}$ to a normalized absolute doubling time, $A b s_{n o r m}$, as $A b s_{\text {norm }}=2^{\text {Dnorm }} D_{\text {control, grand. }} D_{\text {control, grand }}$ is the grand mean of all control doubling times run in the particular experimental series, removing plate and batch bias and allowing direct comparison across experiments. We estimated the adaptation achieved as $A_{a b s}=A b s_{\text {norm }, t}-A b s_{\text {norm }, 0}$. We fitted a Loess regression (span, $\alpha=0.6$; tricubic weighting) to each adaptation trajectory $\left(A_{a b s}, G\right)$ and extracted the Loess estimated $A_{a b s, \text { loess, }}$ at each generation, $G_{s}$, using the function approx (the linear method) in $\mathrm{R}$. We estimated the potential adaptation as $P=A b s_{n o r m, 0, k}-A b s_{n o r m, 0, n o}$ stress. $A b s_{n o r m, 0, k}$ is 
the normalized absolute doubling time of founder $\left(t_{0}\right)$ population in environment $k$; $A b s_{n o r m, 0, n o}$ stress is the normalized absolute doubling time of founder $\left(t_{0}\right)$ populations in absence of stress. We estimated the fraction of the possible adaptation that had been realised at each time point $t$, as $P_{R}=A_{\text {abs,loess }} / P$. $P_{R}$ is reported in text for paraquat adapting populations at specific time points.

Converting cell doubling times into selection coefficients: We estimated selection coefficients for variants driving the observed change in cell doubling time as in (Gjuvsland et al., 2016). By assuming that the change in cell division time imposed by a variant is the only parameter driving change in cell doubling time, we obtain the equation:

$s=\ln (2)\left(\frac{\tau_{\text {founder }}}{\tau_{1}}-1\right)$,

where $s$ is the per generation selection coefficient for a genotype with a cell division time equal to the measured cell doubling time, and $\tau_{1}$ and $\tau_{\text {founder }}$ represent the cell doubling times $\left(D_{\text {norm}}\right)$ of the focal population and the founder population, respectively. We show selection coefficients as the right $y$-axis data in Figure $1 \mathrm{~F}$ and Figure S2C.

\section{RNA sequencing to measure SOD1, SOD2 and CCP1 expression}

Cell populations were pre-cultivated for two consecutive $72 \mathrm{~h}$ batch growth cycles on SC medium, pre-cultures were subsampled and subsamples were transferred to medium w. and w/o paraquat using a Singer Rotor pinning robot (as above). We then sampled cells proliferating in absence of paraquat over one batch growth cycle (72h), and cells proliferating in presence of paraquat over three consecutive batch growth cycles (72h). We also released cells from paraquat stress after three batch growth cycles and sampled them over the following growth cycle in absence 
paraquat. Cells in the first batch cycle were sampled immediately (10-15s) after transfer (stationary phase/transfer), after $0.75 \mathrm{~h}$ (lag phase), 1.5h (lag/exponential phase transition), 5h (mid exponential phase), 20h (late exponential phase) and 25h (exponential/stationary phase transition). Cells in batch cycle two were sampled $0.75 \mathrm{~h}$ after transfer, and cells recovering from three cycles of paraquat exposure were sampled immediately, 0.75 , and $5 \mathrm{~h}$ after transfer. To generate each sample, we cultivated 1536 separate cell populations on a single cultivation plate. Cells to be harvested at early time-points ( $<5 \mathrm{~h}$ after transfer) were cultivated in 6144 colony format, to account for the few cells in each colony at these time point. All samples corresponding to the same batch cycle were cultivated in parallel. We also, in parallel, tracked the growth of non-sampled colonies on two 1536 colony plates to establish in which growth phase each sample was taken. We generated samples by harvesting all colonies on a plate by pouring $5 \mathrm{~mL}$ of medium, w. or w/o paraquat, on top of the solid medium of each plate to be sampled. We scraped off the 1536 or 6144 colonies on the plate with a sterile plastic rake into the liquid medium, pooling all cells on the plate. Cells were collected by pouring the liquid off, pelleting at $12000 \mathrm{G}\left(2 \mathrm{~min}\right.$ in $4^{\circ} \mathrm{C}$ ), re-suspension in RNAlater (Sigma Aldrich; R0901) and storage at $4{ }^{\circ} \mathrm{C}$. We extracted RNA from all the stored samples in parallel, first diluting the RNAlater solution with an equal volume of PBS and then pelleting cells at $5000 \mathrm{G}\left(5 \mathrm{~min}, 4^{\circ} \mathrm{C}\right)$. Cells were lysed by adding $600 \mu \mathrm{L}$ of acid washed $0.5 \mathrm{~mm}$ beads and homogenization in a FastPrep homogenizer (three rounds a $40 \mathrm{~s}$ at $6 \mathrm{~m} \mathrm{~s}^{-}$ ${ }^{1}$ separated by 1 min on ice). RNA quality was determined using a Tapestation 2200 and Nanodrop (threshold; $\mathrm{ABS}_{260 / 280}>2.2$ and RINe $>8$ ). RNA sequencing was performed at SciLife (Stockholm, Sweden) using the Illumina TruSeq Stranded mRNA kit and a NovaSeq 6000 S4. RNA reads were checked for contamination 
using FastQ Screen (Wingett \& Andrews, 2018). Filtered reads were aligned to the YPS128 reference genome using STAR (Dobin et al., 2013), and optical duplicates were marked with Picard-tools. The abundance of the SOD1, SOD2 and CCP1 transcripts was quantified with featureCounts from the subread package across all samples (Liao, Smyth, \& Shi, 2014). We normalized their read counts as fragments per kilobases per million reads, using the DESeq2 package for R (Love, Huber, \& Anders, 2014). The normalized read counts for SOD1, SOD2 and CCP1 are reported in Supplementary Data S20 and shown as $y$-axis data in Figure 1B.

\section{DNA Sequencing of Evolving Cell Populations}

Long read (PacBio) sequencing: To allow accurate calling of mutations, we first de novo assembled the genome of the sample of the founder strain YPS128 used in adaptation experiments. The total genomic DNA was extracted from a founder population cultivated overnight in background medium, using a standard phenolchloroform protocol. We sequenced the genome on a PacBio RS II instrument using the P4-C2 chemistry. Additional PacBio sequencing data of the same YPS128 genotype were incorporated from and older assembly (Yue et al., 2017). A total of 9 SMRT cells were used in the assembly that resulted in 1352628 reads, corresponding to approximately $205 x$ genome coverage. We ran the de novo assembly using the hierarchical assembly protocol RS_HGAP_Assembly3.3 with an expected genome size of $12 \mathrm{Mb}$. Data were deposited at Sequencing Read Archive (SRA), accession number PRJNA622836.

Very long read (Oxford nanopore) sequencing: To exclude confounding effects of very early mtDNA changes, i.e. during freezing, thawing and the first round of paraquat cultivation, we thawed and subsampled frozen lawn cells (A7 lawn position) 
and cultivated these in the presence of paraquat until stationary phase. DNA was extracted using Qiagen Genomic-tip 100/G DNA extraction kit. We sequenced the extracted DNA with reads sufficiently long to exclude confounding effects of the highly repetitive nature of yeast mtDNA. Libraries for Oxford Nanopore sequencing were prepared using 1D Native barcoding genomic DNA with the EXP-NBD104 and SQK-LSK108kit. The flowcell version was FLO-MIN106 The raw nanopore reads were basecalled by guppy (v2.1.3) with a minimal quality score cutoff of 5 (options: -qscore_filtering --min_qscore 5). For all basecalled reads that passed the quality filter, demultiplexing was further performed by guppy with the help of the guppy_reads_classifier.pl from LRSDAY (v1.3.1)(Yue \& Liti, 2018). The demultiplexed reads were processed by LRSDAY (v1.3.1) for adapter trimming, reads downsampling (downsampled to 50X coverage), de novo assembly, assembly polishing, assembly scaffolding, and dotplot visualisation. Reads mapped to the assembled mtDNA data are shown in Figure S3C. We deposited data at Sequencing Read Archive (SRA), accession number PRJNA622836.

Resequencing of adapted populations and populations released from selection: We thawed and subsampled frozen chronological record populations and cultivated cells in liquid medium in presence of the stressor overnight (24h). DNA was extracted using a modified protocol of the Epicentre MasterPure Yeast DNA Purification Kit. Pool sequencing was performed at SciLife (Stockholm, Sweden), using Illumina HiSeq2500, 2x126bp. Libraries were prepared using the Nextera XT kit to accommodate the low DNA yield from small cultures. At least two founder controls were included in each flow cell.

Calling de novo point mutations: Sequenced reads were quality-trimmed and nextera transposase sequences were removed with TrimGalore (v.0.3.8). Reads were 
mapped to the YPS128 pacbio assembly (see above) using BWA MEM (v.0.7.7r441). PCR and optical duplicates were flagged using Picard-tools (v.1.109 [1716]). Base alignment quality scores were calculated using samtools calmd (v.0.1.18 [r982:295]) and variants were called using Freebayes (v0.9.14-8-g1618f7e). All alleles were reported regardless of frequency or genotype model since the populations are a pooled set of individuals. Variants were annotated using SnpEFF (v.3.6c). Variants below a quality score of 20 and variants present in the sequenced founder samples were filtered out. Data were deposited at Sequencing Read Archive (SRA), accession number PRJNA622836. Data is reported in Supplemental Information Data S10 and visualized in Figures S5A and S5B.

Calling aneuploidies: Aneuploidies were called using a sliding, non-overlapping $200 \mathrm{bp}$ window coverage of reads mapped. Reads with a MAPQ of $<1$ were not counted. Window coverage ratio was calculated as:

$$
\log _{2}\left(\frac{k w_{i}}{w_{\text {founder }, i}}\right)
$$

where $w_{n}$ is the depth of coverage of mapped reads in each $200 \mathrm{bp}$ window, $\mathrm{i}, w_{\text {founder }}$ is the depth of coverage of each $i$ in a founder sequenced in the same flow cell and

$$
k=\sum_{i=1}^{G} D_{\text {founder }} / \sum_{i=1}^{G} D_{\text {sample }}
$$

where $G$ is the YPS128 genome size and $D$ the depth of coverage for each nucleotide. Aneuploidies were called determining the median $\log _{2}$ window coverage for each chromosome. Data are shown in Figure 4A and Figures S5A and S5C, and reported in Supplemental Information Data S11 and S12.

Calling mtDNA copy number change: mtDNA copy number was calculated for each sample using a sliding, non-overlapping window of $1 \mathrm{kB}$. The mtDNA copy number relative euploid nuclear genome ratio was calculated for each window as: 


$$
\log _{2}\left(\frac{w_{i}}{w_{\text {median,euploid }}}\right)
$$

where $w_{\text {median,euploid }}$ is the median of all $1 \mathrm{kB}$ windows of the nuclear genome, excluding chromosomes with detected aneuploidies. We estimated the median absolute number of mtDNA molecules across all windows, assuming one copy of the nuclear genome and assuming no sequencing bias for mitochondrial DNA, as

$$
2^{\operatorname{median}\left(\log _{2}\left(\frac{w_{i}}{w_{\text {median,euploid }}}\right)\right)}
$$

These are shown as the left y-axis in Figures 2C, 3C, 3E and Figure S5A, and are reported in Supplemental Information Data S13, S14 and S15.

The right $y$-axis in Figure 3F shows the replenishment of deleted mtDNA segments. An average of the copy number of the $1 \mathrm{kB}$ windows of the end point $\left(t_{10}\right)$ of the relaxation experiment was calculated for each population. We excluded $1 \mathrm{kB}$ windows with a copy number above the average copy number $\left(t_{10}\right)$ to only visualize the change in deleted segments. The average of the deleted segments, defined as below the average copy number at the end point, are shown as red lines.

\section{Simulating Evolving Cell Populations}

Cell population parameters: To generate simulated adaptation trajectories based on empirical effect sizes and mutation rates of point mutations and aneuploidies, we used an individual-based model implemented in Python (Gjuvsland et al., 2016). We repeated each simulation $1152 x$, to match the number of empirically observed adaptation trajectories. We started from a haploid, isogenic founder population that was subsampled at the end of each batch phase to found the next cultivation cycle. Population parameters describe population size at the start of each batch cycle $(N)$, number of cell divisions before subsampling in each batch cycle $\left(M_{t}\right)$ and total 
number of batch cycles ( $n=50$ cycles). When the total population size reached $2^{M t} N$ cells, $N$ cells were sub-sampled randomly to found the next cycle. $N$ was set to equal the approximate mean across all empirical sub-samplings. $M_{t}$ was set to equal the mean (across populations) empirical measure in each batch cycle $t$. Each cell divided 12 times before it died. Mating, meiosis, sporulation or ploidy change were not allowed and there was no population structure.

Mutation rate parameters: All cells began as identical, haploid founder cells. Cells had 4947 nuclear encoded protein genes, and 16 chromosomes specified by the sequenced reference genome (R64-1-1). Cells had no mitochondrial genome. Essential genes were not included. Cells independently and randomly acquired nuclear genome mutations as chromosome duplications and point mutations in protein coding genes at the end of each cell division. Mutation rates were constant, equal for all genomes, for all chromosomes and for all nucleotide sites. Chromosomes and nucleotide sites were only allowed to mutate once. Sites on new chromosomes do not mutate. Chromosome duplications occur at rate, $\mu=4.85^{*} 10^{-5}$ duplications/cell division. Point mutations occurred at rate $\mu=0.33^{*} 10^{-9}$ point mutations/bp/division (Y. O. Zhu, Siegal, Hall, \& Petrov, 2014).

Mutation effect size parameters: We tracked the mutations of each cell, its reproductive age, and its cell division time. Mutations and cell division time was passed on to daughter cells. Cells began at a cell division time equal to the founder population doubling time. Change in cell division time was affected by mutations only and mutations only affected cell division time. Because chromosome duplication and loss-of-gene function point mutations are the most common drivers of experimental adaptation (Chevereau et al., 2015), we approximated these as the only sources of change in cell division time. We estimated the cell division effect size of chromosome 
duplications as described above. We estimated the cell division effect size of point mutations by downloading the SIFT yeast database (http://sift-db.bii.a-star. edu.sg/public/Saccharomyces_cerevisiae/EF4.74/) and extracting all possible stop gain base changes and nonsynonymous mutations, with attached SIFT scores (Vaser, Adusumalli, Leng, Sikic, \& Ng, 2016). All stop gain base changes and all nonsynonymous mutations with a SIFT score $<0.05$ affected cell division time with an effect size equal to the population doubling time, $D_{r}$, effect of the corresponding gene deletion (see above). Reproductive age was not allowed to affect cell division time. There were no cell-cell interactions. We assumed that cell doubling time in stress in the relevant evolutionary time span cannot become shorter than the measured mean founder cell doubling time in absence of stress, $D_{\text {founder,no stress. }}$. We implemented the well documented (Chou, Chiu, Delaney, Segrè, \& Marx, 2011; Khan, Dinh, Schneider, Lenski, \& Cooper, 2011) diminishing return of mutations with increasing fitness, by letting a mutation, $m$, define the cell division time in cells with the mutation, $D_{m}$, as:

$D_{m}=k\left(D_{G}-D_{\text {founder,no stress }}\right)+D_{\text {founder,no stress }}$

$D_{G}$ is the cell division time of the genotype before the mutation occurred. $k$ is defined as:

$$
k=\max \left(\frac{2^{D_{r}} D_{\text {founder }, \text { stress }}-D_{\text {founder }, \text { no stress }}}{D_{\text {founder }, \text { stress }}-D_{\text {founder }, \text { no stress }}}, 0\right)
$$

$D_{\text {founder,stress }}$ is the measured mean doubling time of the founder in presence of the simulated stress. No other form of epistasis was included. We recorded population averages for cell division times at the end of each batch cycle, and plotted it as a 
function of mean evolutionary time (total population doublings). We show the simulation results in Figure 1D.

\section{Quantitative PCR of mtDNA Genes in Evolving Cell Populations}

To track the copy number dynamics of mtDNA genes in evolving populations, we performed quantitative PCR (qPCR) on the frozen chronological samples from a subset of populations (A4, A7, A8, A9, B5, B8, B12, D1). Frozen chronological record was revived in $3 \mathrm{~mL}$ liquid background media supplemented with $400 \mu \mathrm{g} / \mathrm{mL}$ paraquat, expanded to stationary phase $(72 \mathrm{~h})$. Cells were harvested and DNA was extracted using a MasterPure Yeast DNA purification kit (Epicentre), as per the manufacturer's instructions. Primers (Supplemental Information Data S19) were designed for each of the protein and rRNA encoding mtDNA genes and for one nuclear control: CDC5. The small size and extreme AT richness of the RNA subunit of RNase $\mathrm{P}(R P M 1)$ prevented the design of working PCR primers for this mtDNA gene. We ran qPCR for duplicates of the entire frozen chronological record of one mtDNA gene in a single run, together with CDC5 controls. Different mtDNA genes were in separate runs. The qPCR was performed using iTaq Universal SYBR Green Supermix (total volume: $20 \mu \mathrm{L}$ ) and run on a Bio-Rad CFX Connect using Bio-Rad Hard-Shell PCR 96-well thin-wall plates sealed with adhesive transparent film. The PCR protocol was: initial denaturation $\left(95^{\circ} \mathrm{C}\right.$; $\left.15 \mathrm{~min}\right)$ followed by 45 cycles of: denaturation $\left(95^{\circ} \mathrm{C} ; 15 \mathrm{~s}\right)$, anneal $\left(60^{\circ} \mathrm{C} ; 30 \mathrm{~s}\right)$, extension $\left.\left(72^{\circ} \mathrm{C} ; 30 \mathrm{~s}\right)\right]$, and a melting curve analysis. We quantified PCR products at the annealing step of each cycle due to the low melting temperature of all PCR products, which follows from extremely low GC\% of the mtDNA. The relative copy number was calculated as $\log _{2}\left(2^{-\left(C t_{m D N A}-C t_{C D C S}\right)_{t}-\left(C t_{m D D N A}-C t_{C D C S}\right)_{0}}\right)$ 
We capped all $\mathrm{Ct}$ values at 30 . We also set $\mathrm{Ct}$ values to 30 for rare $(5.6 \%)$ sample replicates where the COX1 primer pair produced non-PCR based background signals. qPCR data are shown in Figure 3D and Figure S4, and report the data in Supplemental Information Data S16. Primers used are reported in Supplemental Information Data S19.

\section{Light and Fluorescence Microscopy of Evolving Cells}

We performed light and fluorescence microscopy on DNA (DAPI) stained $\rho^{+}$ (founder, WT), $\rho^{-}$(population A7 at $\left.t_{50}\right), \rho^{--}$(population B8 at $t_{50}$ ) and $\rho^{0}($ mip $1 \Delta$; lacking the mitochondrial DNA polymerase) cells to validate that $\rho^{--}$cells indeed contain mitochondrial DNA. We cultivated cell populations overnight in liquid background medium, diluted pre-cultures in fresh media to $\mathrm{OD}_{600}=0.3$ and incubated with agitation in $15 \mathrm{~mL}$ growth tubes until $\mathrm{OD}_{600}=0.6$. Cells were pelleted by centrifugation at $3000 \mathrm{~g}$ for $1.5 \mathrm{~min}$. The supernatant was discarded and cells were suspended in $1 \mathrm{~mL}$ of ice cold $70 \% \mathrm{EtOH}$. Cells were fixated by incubation in RT for $5 \mathrm{~min}$. The cells were then pelleted again by centrifugation, washed in non-ionic water and re-suspended in PBS (Fisher Bioreagents BP2944-100) solution. DNA was stained with DAPI (D3571, Merck) at $50 \mathrm{ng} / \mathrm{mL}$ just before imaging. Images were acquired using a Zeiss Axio Observer Z1 Inverted microscope with PlanApochromat 100x/1.40 Oil DIC M27 objective and AxioCam MR R3 camera. Data are shown in Figure S6B.

\section{Electron Microscopy of Evolving Cells}

We measured mitochondrial dynamics during $\mathrm{O}_{2}^{-}$stress by electron microscopy of cells before $\mathrm{O}_{2}^{-}$stress, after $5 \mathrm{~h}(\sim 1$ cell doubling $)$ of $\mathrm{O}_{2}^{-}$stress, during chronic $\mathrm{O}_{2}^{-}$ 
stress $\left(A 7, t_{50}\right)$ and $5 \mathrm{~h}$ after release from chronic $0_{2}^{-}$stress $\left(A 7, t_{50}\right)$. Frozen stocks were revived by transfer to solid background medium (with or without paraquat) and cells were cultivated for $5 \mathrm{~h}$ ( 1 population doubling). Cells were harvested by rinsing the plate with $5 \mathrm{~mL}$ liquid background media (with or without paraquat), suspended by stirring with a plastic spreader and pelleted by centrifugation at $600 \mathrm{~g}$ for $1.5 \mathrm{~min}$. Pelleted cells were frozen under high pressure using a Wohlwend Compact 03 (M. Wohlwend $\mathrm{GmbH}$, Sennwald, Switzerland). Freeze substitution was performed in a Leica EM AFS2 (Leica Microsystems, Vienna, Austria) by incubating the cells with $2 \%$ uranyl acetate dissolved in $10 \%$ methanol and $90 \%$ acetone for 1 hour at $-90^{\circ} \mathrm{C}$ (Hawes, Netherton, Mueller, Wileman, \& Monaghan, 2007). Freeze substituted cells were washed $(2 \mathrm{x})$ in $100 \%$ acetone and the temperature was raised $2.9^{\circ} \mathrm{C}$ per hour to $-50^{\circ} \mathrm{C}$. Cell pellets were broken into smaller pieces to improve resin infiltration. Infiltration of cells was performed using a ladder of Lowicryl HM20 (Polysciences, Warrington, PA) diluted in decreasing acetone concentrations $(1: 4,2: 3,1: 1,4: 1)$ followed by three changes in pure Lowicryl. Each step lasted $2 \mathrm{~h}$. The resin was polymerized with UV light, first for $72 \mathrm{~h}$ at $-50^{\circ} \mathrm{C}$ and then for $24 \mathrm{~h}$ at room temperature. Resin embedded blocks were sectioned in $70 \mathrm{~nm}$ ultra-thin sections using a Reichert-Jung Ultracut E Ultramicrotome (C. Reichert, Vienna, Austria) equipped with an ultra $45^{\circ}$ diamond knife (Diatome, Biel, Switzerland). Sections were collected on copper grids coated with $1 \%$ formvar and stained with $2 \%$ uranyl acetate and Reynold's lead citrate (Reynolds, 1963). Stained sections were imaged at $120 \mathrm{kV}$ using a Tecnai T12 microscope (FEl Co., Eindhoven, The Netherlands) and a Ceta CMOS16 camera. The IMOD package (Kremer, Mastronarde, \& Mclntosh, 1996) was used for quantification of 100 cell sections per sample. To validate quantifications, a large subset of images was analyzed by blind-test by a 
second person. Conflicting quantifications were discarded. We report this data in Supplemental Information Data S17 and S18. We show these data in Figure 2A.

\section{Confocal Microscopy of Evolving Cells}

We tracked mitochondrial dynamics during $\mathrm{O}_{2}^{-}$stress by confocal microscopy of fluorescently labelled (Cox4-EGFP) mitochondria in cells before $\mathrm{O}_{2}^{-}$stress, after $7 \mathrm{~h}$ ( 1 doubling) of $\mathrm{O}_{2}^{-}$stress, after $79 \mathrm{~h}$ ( 1 growth cycle +1 doubling) of $\mathrm{O}_{2}^{-}$stress and $7 \mathrm{~h}$ after release from $144 \mathrm{~h}$ ( 2 growth cycles) of $\mathrm{O}_{2}^{-}$stress. We isolated 3 transformants and ensured that their respiratory growth was normal by spot-assays on glycerol medium. Transformants were cultivated with or without paraquat for $0 \mathrm{~h}$ or $72 \mathrm{~h}$ in liquid medium, diluted stationary phase cultures in fresh media with or without paraquat to $\mathrm{OD}_{600}=0.3$ and incubated diluted cultures with agitation until $\mathrm{OD}_{600}=0.6$ (exponential phase). Cells were pelleted by centrifugation at $3000 \mathrm{~g}$ for $1.5 \mathrm{~min}$ and washed in MQ water, centrifuged again and suspended in PBS. Cells were fixed by incubation at room temperature with $3.7 \%$ formaldehyde, washed 3 times in PBS, suspended in ProLong Diamond mounting media and directly mounted on slides and imaged in the microscope. Z-stacks of cells were acquired using a Zeiss Axio Observer LSM 700 inverted confocal microscopy with a Plan-Apochromat 63x/1.40 Oil DIC M27. The signal was averaged between 4 frames to reduce noise. Z-stacks were color-coded according to Z-dimension (slice) using using Temporal-Color Code in Fiji/lmageJ v. 1.52. We show the data in Figure 2B and Figure S3B.

Resource Availability: Sequence data that support the findings of this study have been deposited in Sequencing Read Archive (SRA) with the accession codes PRJNA622836, PRJNA622836 and PRJNA622836. The simulation code can be 
found at (https://github.com/HelstVadsom/GenomeAdaptation.git). The authors declare that all other data supporting the findings of this study are available within the paper (Supplemental Information Data S1-S20, which can be previewed at https://data.mendeley.com/datasets/mvx7t7rw2d/draft?a=95381e47-dc80-47af-85abe0478912a209).

Materials Availability: All unique strains and populations generated in this study are available from the Lead Contact without restriction.

\section{SUPPLEMENTAL INFORMATION}

Fig S1-S6

Table S1

Data S1-S20 (https://data.mendeley.com/datasets/mvx7t7rw2d/draft?a=95381e47dc80-47af-85ab-e0478912a209) 


\section{SUPPLEMENTARY FIGURES}

\section{Figure S1. Design of Adaptation Experiments}

We adapted 1152 homogeneous, haploid yeast populations to paraquat $\left(0_{2}^{-}\right.$stress $)$ and seven non-mitochondrial challenges (Table S1) over 50 cycles $\left(t_{1}-t_{50}\right)$ of expansion and subsampling. We counted cells $(n=1)$ in each population at 20 min intervals and extracted cell doubling times, $D$, at the mid-exponential phase and counted cell generations, $G$, as the number of population doublings from the start to end of each batch cycle. We stored batch cycles $0-5,7,9,12,15,20,25,30,35,40$, 45 and 50 of the same 96 populations as a frozen record. We revived and reanalyzed these $(n=6)$ in a randomized design to accurately capture the adaptation kinetics for 768 populations. We extracted cell doubling times, $D$, and $\log _{2}$ normalized these to those of many founder controls, $D_{\text {norm }}$. We subtracted the $D_{\text {norm }}$ before stress exposure, $D_{\text {norm, } 0}$, to estimate the adaptation achieved, $A$.

\section{Figure S2. Adaptation to $\mathrm{O}_{2}^{-}$Stress is Swift and Reversible}

(A) Cell doubling time $\left(D_{\text {norm }}\right)$ as a function of generations of evolution $(G)$ for 9216 cell populations adapting to $\mathrm{O}_{2}^{-}$stress or one of seven non-mitochondrial challenges (Supplemental Information Table S1). Lines: 1152 populations $(n=1)$. Inset: zoom-in on first cycles of paraquat adaptation. (B) Loss of adaptation ( $y$-axis, $A)$ to $\mathrm{O}_{2}^{-}$, arsenic and glycine stress after release from the stress $(x$-axis, generations after release, $G_{r}$ ). Colored lines: mean of 96 populations (each at $n=5$ ). Shade: S.E.M. Cell populations were released from stress after reaching $70-90 \%$ of their endpoint $\left(t_{50}\right)$ adaptation. $(C)$ Cell doubling time $(h)$ difference between adapted and founder populations, in the absence of stress. Mean of $96 \mathrm{O}_{2}^{-}$, arsenic and glycine adapted populations (as in Figure S2B) are shown. Right $y$-axis: Cell doubling time difference 
converted into a selection coefficient, s. Mean of 96 populations (each at $n=5$ ) is shown. Error bars: S.E.M.

Figure S3. $\mathrm{O}_{2}^{-}$stress Leads to Rapid, Reversible Mitochondrial Fragmentation

(A) Confocal microscopy of yeast cells with a Cox4-GPF mitochondrial tag, before (left), after $7 \mathrm{~h}$ (center left) and after 1 growth cycle $(72 \mathrm{~h})+7 \mathrm{~h}$ (center right) of $\mathrm{O}_{2}^{-}$ stress and $7 \mathrm{~h}$ after release from 2 growth cycles (right) of $\mathrm{O}_{2}^{-}$stress. Color: $\mathrm{z}-$ dimension (yellow=front, black=back). Two Cox4-GFP transformants, isolated independently from that in Figure 2B, are shown. (B) Virtually all mitochondria fragments contain mtDNA during early $\mathrm{O}_{2}^{-}$stress. Micrographs: confocal microscopy of yeast cells after $7 \mathrm{~h}$ of $\mathrm{O}_{2}^{-}$stress. The Cox4-GFP signal in the inner mitochondrial membrane (left), mtDNA stained with DAPI (middle) and the merging of both these images (right), are shown. (C) The mitochondrial DNA is retained intact at pre-stres copy number after 3 generations (1 growth cycle) of $\mathrm{O}_{2}^{-}$stress. $y$-axis, median mtDNA coverage, in $0.5 \mathrm{~kb}$ windows, relative to that of the haploid nuclear genome. $x$-axis: mtDNA position. We exposed a founder cell population to $\mathrm{O}_{2}^{-}$stress for a single growth cycle $(72 \mathrm{~h})$ and sequenced the extracted DNA with long-read sequencing.

\section{Figure S4. mtDNA Deletions Drive the Early $\mathrm{O}_{2}^{-}$Adaptation}

Early $\mathrm{O}_{2}^{-}$stress causes segmental mtDNA deletions. Circle: mtDNA (77kB) before $\mathrm{O}_{2}^{-}$ stress adaptation, with genes, known origins of replication and position (numbers) indicated. Diagrams: mtDNA copy number (left $y$-axis, qPCR, purple line) of each protein and rRNA encoding genes and $\mathrm{O}_{2}^{-}$adaptation (right $y$-axis, $A$, green line) in populations A4, A9, A8, B8, B5, B12 and D1 over generations of $\mathrm{O}_{2}^{-}$exposure ( $x$ - 
axis, $G_{s}$ ). Error bars: S.E.M. $(n=2)$. Colored fields: deleted mtDNA segments, with concerted copy number change.

\section{Figure S5. Nuclear Genome Evolution During Chronic $0_{2}^{-}$Stress}

We short-read sequenced 44 endpoint $\left(t_{50}\right)$ populations adapted to chronic $\mathrm{O}_{2}^{-}$stress and called de novo point mutations and chromosome duplications relative to the founder genome assembly. (A) mtDNA deletion and chromosome II, III and V duplications recur across populations adapted to chronic $\mathrm{O}_{2}^{-}$stress, but nuclear genes with point mutations rarely do. Upper $x$-axis: Number of populations in which a gene carries de novo point mutations, a chromosome is duplicated or a mtDNA segment is deleted. Dotted line: number of sequenced populations. Lower $x$-axis (grey line): For genes containing SNPs, the line shows the mean allele frequencies of SNPs in the gene, for chromosome or mtDNA copy number variations the line shows the mean sequence coverage across the chromosome or mtDNA relative to that of the haploid nuclear genome. $y$-axis: Genes with point mutations, chromosomes with duplications and mtDNA. Bar color: Type of variation. (B) Adaptation to $\mathrm{O}_{2}^{-}$stress (right $y$-axis, green line, $A$, shade: S.E.M. $(n=6)$ precedes point mutations (left $y$-axis, non-green lines, allele frequency). $x$-axis: Generations $\left(G_{s}\right)$ of adaptation to $\mathrm{O}_{2}^{-}$stress. Panels: Sequenced populations (A7, A8, B5, B8, and B12). Line color: variant type. Variants pre-dating adaptation, supported by few $(<10)$ reads or $(<2)$ time points or shared across environments $(>2)$ were filtered out. $(C)$ Chromosome duplications occur in the second phase of $\mathrm{O}_{2}^{-}$stress adaptation. Panels: five populations (A7, A8, B5, B8, and B12). Left y-axis (non-green lines): Chromosome copy number ( $\log _{2}$ of median sequence coverage across the chromosome relative to that of the median haploid nuclear genome). Color: 
chromosome (II=blue, III=red and $\mathrm{V}=$ =yellow). Right $y$-axis (green line): $\mathrm{O}_{2}^{-}$adaptation, A. Shade: S.E.M. $(n=6)$. Broken lines: no data.

\section{Figure S6. Mitochondrial Genome Evolution During Chronic $\mathbf{0}_{2}^{-}$Stress}

(A) Sequenced populations $(n=44)$ adapted $\left(t_{50}\right)$ to chronic $0_{2}^{-}$stress were classified (colour) as $\rho^{+}$(mtDNA intact, $\left.n=1\right), \rho^{-}(6-30 \mathrm{~kb}$ mtDNA segments retained, $n=18)$ and $\rho^{--}(<2 \mathrm{~kb}$ mtDNA segments retained, $n=25)$. $y$-axis: mtDNA copy number (median coverage in $0.5 \mathrm{~kb}$ windows relative to haploid nuclear genome). $x$-axis: mtDNA position. Below: gene positions. Read map: a zoom-in on a 300bp mtDNA stretch where mtDNA sequence reads in the $\rho^{--}$B8 population maps. (B) $\rho^{--}$cells with low mtDNA sequence coverage retain very short $(<300)$ mtDNA segments. Micrographs: Light (top; DIC) and fluorescence (bottom) microscopy of a DAPI stained DNA in $\rho^{--}$(B8 at $\left.t_{50}\right), \rho^{+}$(founder), $\rho^{-}$(A7 at $\left.t_{50}\right)$, and true $\rho^{0}$ (mip1 1 cells; lacking the yeast mitochondrial DNA polymerase) cells. Insets: Zoom-in on indicated $\rho^{--}$and true $\rho^{0}$ cells. Note that $\rho^{--}$cells contain cytoplasmic (mitochondrial) DNA while true $\rho^{0}$ cells do not. (C) Chromosome duplications do not explain the complete loss of respiratory growth. We backcrossed $(x 3)$ cells adapted to chronic $\mathrm{O}_{2}^{-}$stress $\left(t_{50}\right)$ to founder cells over consecutive meiotic generations and compared the doubling time of 2-3 clones of 3rd generation offspring w. and w/o duplicated chromosome II, III and V, under respiratory (glycerol) growth. $y$-axis: Doubling time $\left(D_{\text {norm }}\right) . x$-axis: cells w. $(+)$ and w/o (-) individual chromosome duplications. Error bars: S.E.M. (n=6). (D) Cell populations becoming $\rho^{--}\left(<2 \mathrm{~kb}, \rho^{--}\right.$red line, $\left.n=25\right)$ after chronic $\mathrm{O}_{2}^{-}$stress $\left(t_{50}\right)$ are less fit than populations becoming $\rho^{-}(6-30 \mathrm{~kb}$, green line, $n=18)$. $y$-axis: $\mathrm{O}_{2}^{-}$adaptation, $A$. Shade: S.E.M. of 18 or 25 populations, each at $n=6$. The shown experiments is an independent repeat of that in Figure 5D. 


\section{REFERENCES}

Allen, J. F. (2017). The CoRR hypothesis for genes in organelles. Journal of Theoretical Biology, 434, 50-57. https://doi.org/10.1016/j.jtbi.2017.04.008

Ammal Kaidery, N., \& Thomas, B. (2018). Current perspective of mitochondrial biology in Parkinson's disease. Neurochemistry International, 117, 91-113. https://doi.org/10.1016/j.neuint.2018.03.001

Anderson, A. P., Luo, X., Russell, W., \& Yin, Y. W. (2020). Oxidative damage diminishes mitochondrial DNA polymerase replication fidelity. Nucleic Acids Research, 48(2), 817-829. https://doi.org/10.1093/nar/gkz1018

Andrukhiv, A., Costa, A. D., West, I. C., \& Garlid, K. D. (2006). Opening mitoK ATP increases superoxide generation from complex I of the electron transport chain. American Journal of Physiology-Heart and Circulatory Physiology, 291(5), H2067-H2074. https://doi.org/10.1152/ajpheart.00272.2006

Bess, A. S., Crocker, T. L., Ryde, I. T., \& Meyer, J. N. (2012). Mitochondrial dynamics and autophagy aid in removal of persistent mitochondrial DNA damage in Caenorhabditis elegans. Nucleic Acids Research, 40(16), 79167931. https://doi.org/10.1093/nar/gks532

Brand, M. D. (2016). Mitochondrial generation of superoxide and hydrogen peroxide as the source of mitochondrial redox signaling. Free Radical Biology and Medicine, 100, 14-31. https://doi.org/10.1016/j.freeradbiomed.2016.04.001

Castello, P. R., Drechsel, D. A., \& Patel, M. (2007). Mitochondria are a major source of paraquat-induced reactive oxygen species production in the brain. The Journal of Biological Chemistry, 282(19), 14186-14193. https://doi.org/10.1074/jbc.M700827200

Chevereau, G., Dravecká, M., Batur, T., Guvenek, A., Ayhan, D. H., Toprak, E., \& Bollenbach, T. (2015). Quantifying the Determinants of Evolutionary Dynamics Leading to Drug Resistance. PLoS Biology, 13(11), e1002299. https://doi.org/10.1371/journal.pbio.1002299

Chou, H.-H., Chiu, H.-C., Delaney, N. F., Segrè, D., \& Marx, C. J. (2011). Diminishing returns epistasis among beneficial mutations decelerates adaptation. Science (New York, N.Y.), 332(6034), 1190-1192. https://doi.org/10.1126/science.1203799

Cochemé, H. M., \& Murphy, M. P. (2008). Complex I is the major site of mitochondrial superoxide production by paraquat. Journal of Biological Chemistry, 283(4), 1786-1798. https://doi.org/10.1074/jbc.M708597200

Crabtree, H. G. (1929). Observations on the carbohydrate metabolism of tumours. The Biochemical Journal, 23(3), 536-545. https://doi.org/10.1042/bj0230536

De Chiara, M., Barré, B., Persson, K., Chioma, A. O., Irizar, A., Schacherer, J., ... Liti, G. (2020). Domestication reprogrammed the budding yeast life cycle. BioRxiv, 2020.02.08.939314. https://doi.org/10.1101/2020.02.08.939314

Dobin, A., Davis, C. A., Schlesinger, F., Drenkow, J., Zaleski, C., Jha, S., ... Gingeras, T. R. (2013). STAR: ultrafast universal RNA-seq aligner.

Bioinformatics, 29(1), 15-21. https://doi.org/10.1093/bioinformatics/bts635

Fang, J., \& Beattie, D. S. (2003). External alternative NADH dehydrogenase of Saccharomyces cerevisiae: a potential source of superoxide. Free Radical Biology \& Medicine, 34(4), 478-488. https://doi.org/10.1016/s08915849(02)01328-X

Ferrucci, L., \& Fabbri, E. (2018). Inflammageing : chronic inflammation and frailty, 15(September). 
Frank, M., Duvezin-Caubet, S., Koob, S., Occhipinti, A., Jagasia, R., Petcherski, A., ... Reichert, A. S. (2012). Mitophagy is triggered by mild oxidative stress in a mitochondrial fission dependent manner. Biochimica et Biophysica Acta Molecular Cell Research, 1823(12), 2297-2310. https://doi.org/10.1016/j.bbamcr.2012.08.007

Gaisne, M., Bécam, A. M., Verdière, J., \& Herbert, C. J. (1999). A "natural” mutation in Saccharomyces cerevisiae strains derived from S288c affects the complex regulatory gene HAP1 (CYP1). Current Genetics, 36(4), 195-200. https://doi.org/10.1007/s002940050490

Giaever, G., Chu, A. M., Ni, L., Connelly, C., Riles, L., Véronneau, S., ... Johnston, M. (2002). Functional profiling of the Saccharomyces cerevisiae genome. Nature, 418(6896), 387-391. https://doi.org/10.1038/nature00935

Gjuvsland, A. B., Zörgö, E., Samy, J. K., Stenberg, S., Demirsoy, I. H., Roque, F., ... Warringer, J. (2016). Disentangling genetic and epigenetic determinants of ultrafast adaptation. Molecular Systems Biology, 12(12), 892. https://doi.org/10.15252/msb.20166951

Gustafsson, A. B., \& Dorn, G. W. (2019). Evolving and expanding the roles of mitophagy as a homeostatic and pathogenic process. Physiological Reviews, 99(1), 853-892. https://doi.org/10.1152/physrev.00005.2018

Gutiérrez, A., Sancho, M., Beltran, G., Guillamon, J. M., \& Warringer, J. (2016). Replenishment and mobilization of intracellular nitrogen pools decouples wine yeast nitrogen uptake from growth. Applied Microbiology and Biotechnology, 100(7), 3255-3265. https://doi.org/10.1007/s00253-015-7273-y

Hawes, P., Netherton, C. L., Mueller, M., Wileman, T., \& Monaghan, P. (2007). Rapid freeze-substitution preserves membranes in high-pressure frozen tissue culture cells. Journal of Microscopy, 226(Pt 2), 182-189.

https://doi.org/10.1111/j.1365-2818.2007.01767.x

Hepple, R. T. (2016). Impact of aging on mitochondrial function in cardiac and skeletal muscle. Free Radical Biology \& Medicine, 98, 177-186. https://doi.org/10.1016/j.freeradbiomed.2016.03.017

Hu, H., Tan, C.-C., Tan, L., \& Yu, J.-T. (2017). A Mitocentric View of Alzheimer's Disease. Molecular Neurobiology, 54(8), 6046-6060. https://doi.org/10.1007/s12035-016-0117-7

Hung, C. H.-L., Cheng, S. S.-Y., Cheung, Y.-T., Wuwongse, S., Zhang, N. Q., Ho, Y.-S., ... Chang, R. C.-C. (2018). A reciprocal relationship between reactive oxygen species and mitochondrial dynamics in neurodegeneration. Redox Biology, 14(July), 7-19. https://doi.org/10.1016/j.redox.2017.08.010

Hyttinen, J. M. T., Viiri, J., Kaarniranta, K., \& Błasiak, J. (2018). Mitochondrial quality control in AMD: does mitophagy play a pivotal role? Cellular and Molecular Life Sciences : CMLS, 75(16), 2991-3008. https://doi.org/10.1007/s00018-018-28437

Johnston, I. G., \& Williams, B. P. (2016). Evolutionary Inference across Eukaryotes Identifies Specific Pressures Favoring Mitochondrial Gene Retention. Cell Systems, 2(2), 101-111. https://doi.org/10.1016/j.cels.2016.01.013

Katajisto, P., Döhla, J., Chaffer, C. L., Pentinmikko, N., Marjanovic, N., Iqbal, S., ... Sabatini, D. M. (2015). Asymmetric apportioning of aged mitochondria between daughter cells is required for stemness. Science, 348(6232), 340-343. https://doi.org/10.1126/science.1260384

Kauppila, T. E. S., Kauppila, J. H. K., \& Larsson, N. G. (2017). Mammalian Mitochondria and Aging: An Update. Cell Metabolism, 25(1), 57-71. 
https://doi.org/10.1016/j.cmet.2016.09.017

Khan, A. I., Dinh, D. M., Schneider, D., Lenski, R. E., \& Cooper, T. F. (2011).

Negative epistasis between beneficial mutations in an evolving bacterial population. Science (New York, N.Y.), 332(6034), 1193-1196.

https://doi.org/10.1126/science.1203801

Kremer, J. R., Mastronarde, D. N., \& McIntosh, J. R. (1996). Computer visualization of three-dimensional image data using IMOD. Journal of Structural Biology, 116(1), 71-76. https://doi.org/10.1006/jsbi.1996.0013

Leadsham, J. E., Sanders, G., Giannaki, S., Bastow, E. L., Hutton, R., Naeimi, W. R., ... Gourlay, C. W. (2013). Loss of cytochrome c oxidase promotes rasdependent ros production from the er resident nadph oxidase, yno1p, in yeast. Cell Metabolism, 18(2), 279-286. https://doi.org/10.1016/j.cmet.2013.07.005

Lemasters, J. J. (2005). Selective Mitochondrial Autophagy, or Mitophagy, as a Targeted Defense Against Oxidative Stress, Mitochondrial Dysfunction, and Aging. Rejuvenation Research, 8(1), 3-5. https://doi.org/10.1089/rej.2005.8.3

Liao, Y., Smyth, G. K., \& Shi, W. (2014). featureCounts: an efficient general purpose program for assigning sequence reads to genomic features. Bioinformatics, 30(7), 923-930. https://doi.org/10.1093/bioinformatics/btt656

Lieber, T., Jeedigunta, S. P., Palozzi, J. M., Lehmann, R., \& Hurd, T. R. (2019). Mitochondrial fragmentation drives selective removal of deleterious mtDNA in the germline. Nature, 570(7761), 380-384. https://doi.org/10.1038/s41586-0191213-4

Liti, G., Carter, D. M., Moses, A. M., Warringer, J., Parts, L., James, S. A., ... Louis, E. J. (2009). Population genomics of domestic and wild yeasts. Nature, 458(7236), 337-341. https://doi.org/10.1038/nature07743

Love, M. I., Huber, W., \& Anders, S. (2014). Moderated estimation of fold change and dispersion for RNA-seq data with DESeq2. Genome Biology, 15(12), 550. https://doi.org/10.1186/s13059-014-0550-8

Macao, B., Uhler, J. P., Siibak, T., Zhu, X., Shi, Y., Sheng, W., ... Falkenberg, M. (2015). The exonuclease activity of DNA polymerase $y$ is required for ligation during mitochondrial DNA replication. Nature Communications, 6, 7303. https://doi.org/10.1038/ncomms8303

Medawar, P. B. (1952). An unsolved problem of biology. HK Lewis \& Co., London.

Nido, G. S., Dölle, C., Flønes, I., Tuppen, H. A., Alves, G., Tysnes, O. B., ... Tzoulis, C. (2018). Ultradeep mapping of neuronal mitochondrial deletions in Parkinson's disease. Neurobiology of Aging, 63, 120-127.

https://doi.org/10.1016/j.neurobiolaging.2017.10.024

Palikaras, K., \& Tavernarakis, N. (2014). Mitochondrial homeostasis: the interplay between mitophagy and mitochondrial biogenesis. Experimental Gerontology, 56, 182-188. https://doi.org/10.1016/j.exger.2014.01.021

Picca, A., Calvani, R., Coelho-Junior, H. J., Landi, F., Bernabei, R., \& Marzetti, E. (2020). Mitochondrial Dysfunction, Oxidative Stress, and Neuroinflammation: Intertwined Roads to Neurodegeneration. Antioxidants, 9(8), 647. https://doi.org/10.3390/antiox9080647

Reddi, A. R., \& Culotta, V. C. (2013). SOD1 integrates signals from oxygen and glucose to repress respiration. Cell, 152(1-2), 224-235.

https://doi.org/10.1016/j.cell.2012.11.046

Reynolds, E. S. (1963). The use of lead citrate at high $\mathrm{pH}$ as an electron-opaque stain in electron microscopy. The Journal of Cell Biology, 17, 208-212. https://doi.org/10.1083/jcb.17.1.208 
Rinnerthaler, M., Buttner, S., Laun, P., Heeren, G., Felder, T. K., Klinger, H., ... Breitenbach, M. (2012). Yno1p/Aim14p, a NADPH-oxidase ortholog, controls extramitochondrial reactive oxygen species generation, apoptosis, and actin cable formation in yeast. Proceedings of the National Academy of Sciences, 109(22), 8658-8663. https://doi.org/10.1073/pnas.1201629109

Schöttker, B., Brenner, H., Jansen, E. H. J. M., Gardiner, J., Peasey, A., Kubínová, R., ... Bobak, M. (2015). Evidence for the free radical/oxidative stress theory of ageing from the CHANCES consortium: a meta-analysis of individual participant data. BMC Medicine, 13, 300. https://doi.org/10.1186/s12916-015-0537-7

Sedlackova, L., \& Korolchuk, V. I. (2019). Mitochondrial quality control as a key determinant of cell survival. Biochimica et Biophysica Acta - Molecular Cell Research, 1866(4), 575-587. https://doi.org/10.1016/j.bbamcr.2018.12.012

Selivanov, V. A., Zeak, J. A., Roca, J., Cascante, M., Trucco, M., \& Votyakova, T. V. (2008). The Role of External and Matrix pH in Mitochondrial Reactive Oxygen Species Generation. Journal of Biological Chemistry, 283(43), 29292-29300. https://doi.org/10.1074/jbc.M801019200

Shpilka, T., \& Haynes, C. M. (2018). The mitochondrial UPR: Mechanisms, physiological functions and implications in ageing. Nature Reviews Molecular Cell Biology, 19(2), 109-120. https://doi.org/10.1038/nrm.2017.110

Sies, H. (2018). On the history of oxidative stress: Concept and some aspects of current development. Current Opinion in Toxicology, 7, 122-126. https://doi.org/10.1016/j.cotox.2018.01.002

Sprenger, H. G., \& Langer, T. (2019). The Good and the Bad of Mitochondrial Breakups. Trends in Cell Biology, 29(11), 888-900.

https://doi.org/10.1016/j.tcb.2019.08.003

Stumpf, J. D., \& Copeland, W. C. (2013). The exonuclease activity of the yeast mitochondrial DNA polymerase $y$ suppresses mitochondrial DNA deletions between short direct repeats in Saccharomyces cerevisiae. Genetics, 194(2), 519-522. https://doi.org/10.1534/genetics.113.150920

Sun, N., Youle, R. J., \& Finkel, T. (2016). The Mitochondrial Basis of Aging. Molecular Cell, 61(5), 654-666. https://doi.org/10.1016/j.molcel.2016.01.028

Tadi, S. K., Sebastian, R., Dahal, S., Babu, R. K., Choudhary, B., \& Raghavan, S. C. (2016). Microhomology-mediated end joining is the principal mediator of doublestrand break repair during mitochondrial DNA lesions. Molecular Biology of the Cell, 27(2), 223-235. https://doi.org/10.1091/mbc.E15-05-0260

Timón-Gómez, A., Sanfeliu-Redondo, D., Pascual-Ahuir, A., \& Proft, M. (2018). Regulation of the stress-activated degradation of mitochondrial respiratory complexes in yeast. Frontiers in Microbiology, 9(JAN), 1-16. https://doi.org/10.3389/fmicb.2018.00106

Turrens, J. F. (1997). Superoxide production by the mitochondrial respiratory chain. Bioscience Reports, 17(1), 3-8. https://doi.org/10.1023/a:1027374931887

van Horssen, J., van Schaik, P., \& Witte, M. (2019). Inflammation and mitochondrial dysfunction: A vicious circle in neurodegenerative disorders? Neuroscience Letters, 710. https://doi.org/10.1016/j.neulet.2017.06.050

Vaser, R., Adusumalli, S., Leng, S. N., Sikic, M., \& Ng, P. C. (2016). SIFT missense predictions for genomes. Nature Protocols, 11(1), 1-9.

https://doi.org/10.1038/nprot.2015.123

Vevea, J. D., Swayne, T. C., Boldogh, I. R., \& Pon, L. A. (2014). Inheritance of the fittest mitochondria in yeast. Trends in Cell Biology, 24(1), 53-60. https://doi.org/10.1016/j.tcb.2013.07.003 
Warringer, J., Zörgö, E., Cubillos, F. A., Zia, A., Gjuvsland, A., Simpson, J. T., ... Blomberg, A. (2011). Trait variation in yeast is defined by population history. PLoS Genetics, 7(6), e1002111. https://doi.org/10.1371/journal.pgen.1002111

Wingett, S. W., \& Andrews, S. (2018). FastQ Screen: A tool for multi-genome mapping and quality control. F1000Research, 7, 1338. https://doi.org/10.12688/f1000research.15931.2

Yang, D., Elner, S. G., Bian, Z.-M., Till, G. O., Petty, H. R., \& Elner, V. M. (2007). Pro-inflammatory cytokines increase reactive oxygen species through mitochondria and NADPH oxidase in cultured RPE cells. Experimental Eye Research, 85(4), 462-472. https://doi.org/10.1016/j.exer.2007.06.013

Yue, J.-X., Li, J., Aigrain, L., Hallin, J., Persson, K., Oliver, K., ... Liti, G. (2017). Contrasting evolutionary genome dynamics between domesticated and wild yeasts. Nature Genetics, 49(6), 913-924. https://doi.org/10.1038/ng.3847

Yue, J.-X., \& Liti, G. (2018). Long-read sequencing data analysis for yeasts. Nature Protocols, 13(6), 1213-1231. https://doi.org/10.1038/nprot.2018.025

Zackrisson, M., Hallin, J., Ottosson, L.-G., Dahl, P., Fernandez-Parada, E., Ländström, E., ... Blomberg, A. (2016). Scan-o-matic: High-Resolution Microbial Phenomics at a Massive Scale. G3 (Bethesda, Md.), 6(9), 3003-3014. https://doi.org/10.1534/g3.116.032342

Zebrowski, D. C., \& Kaback, D. B. (2008). A simple method for isolating disomic strains of Saccharomyces cerevisiae. Yeast (Chichester, England), 25(5), 321326. https://doi.org/10.1002/yea.1590

Zhu, J., Zhang, Z.-T., Tang, S.-W., Zhao, B.-S., Li, H., Song, J.-Z., ... Xie, Z. (2019). A Validated Set of Fluorescent-Protein-Based Markers for Major Organelles in Yeast (Saccharomyces cerevisiae). MBio, 10(5), 1-19. https://doi.org/10.1128/mBio.01691-19

Zhu, Y. O., Siegal, M. L., Hall, D. W., \& Petrov, D. A. (2014). Precise estimates of mutation rate and spectrum in yeast. Proceedings of the National Academy of Sciences of the United States of America, 111(22), E2310-8. https://doi.org/10.1073/pnas.1323011111

Zou, X., Ratti, B. A., O’Brien, J. G., Lautenschlager, S. O., Gius, D. R., Bonini, M. G., \& Zhu, Y. (2017). Manganese superoxide dismutase (SOD2): is there a center in the universe of mitochondrial redox signaling? Journal of Bioenergetics and Biomembranes, 49(4), 325-333. https://doi.org/10.1007/s10863-017-9718-8 
bioRxiv preprint doi: https://doi.org/10.1101/2020.11.20.391110; this version posted November 20, 2020. The copyright holder for this

preprint (which was not certified by peer review) is the author/funder. All rights reserved. No reuse allowed without permission.
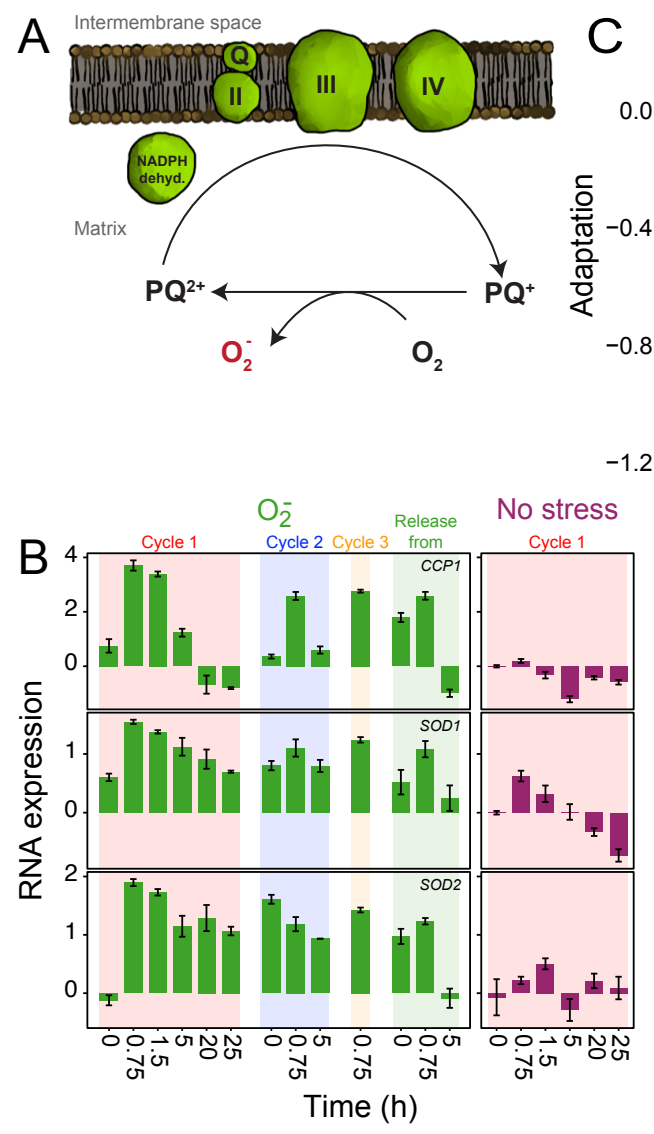
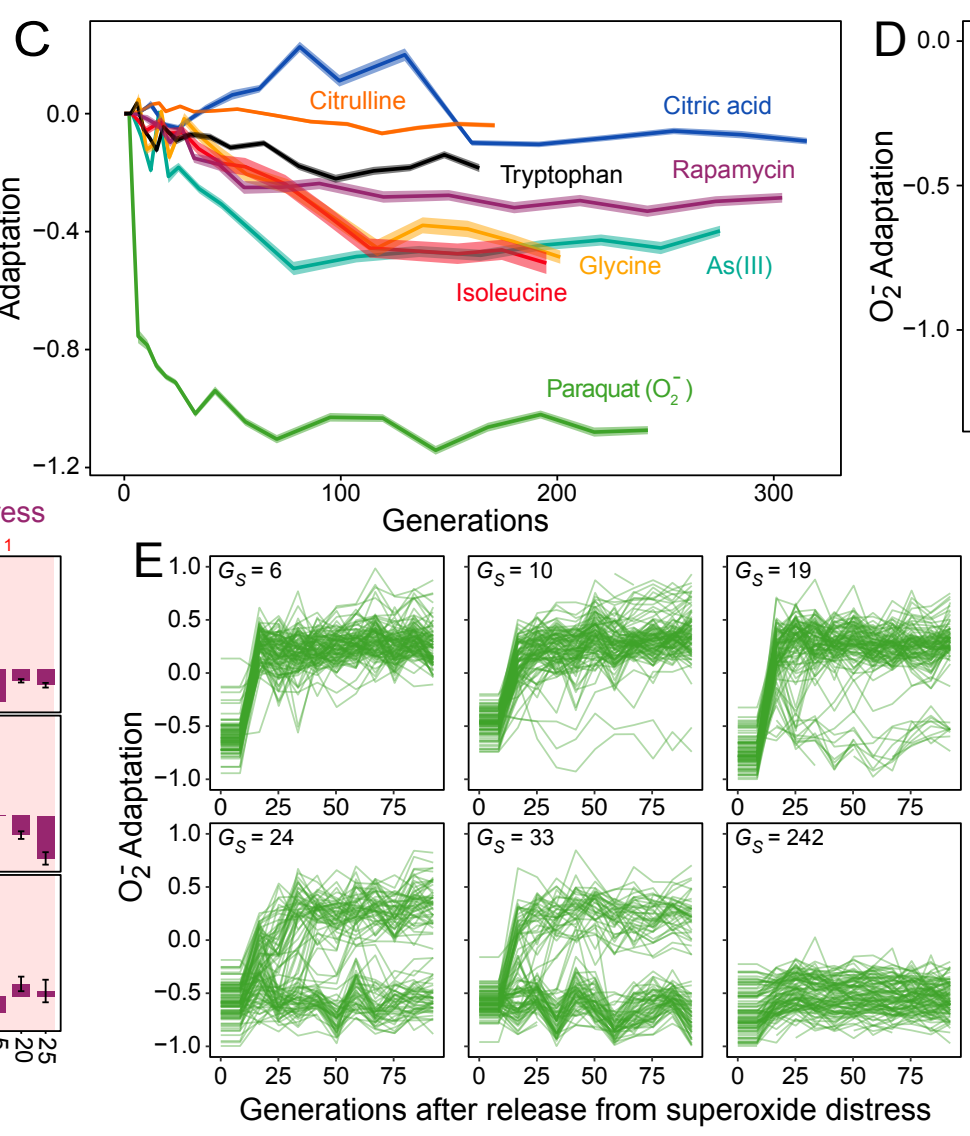
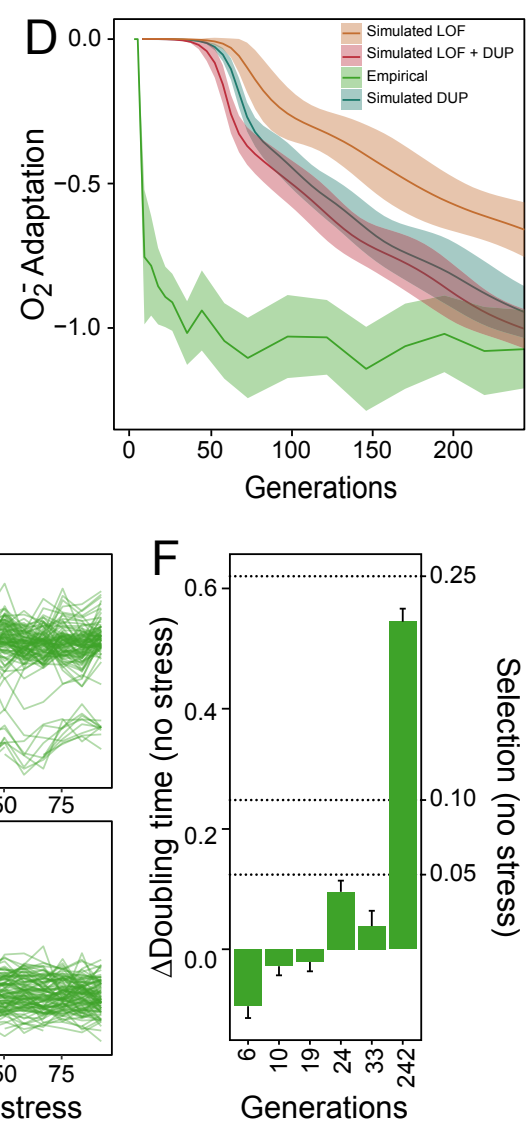

Figure 1 

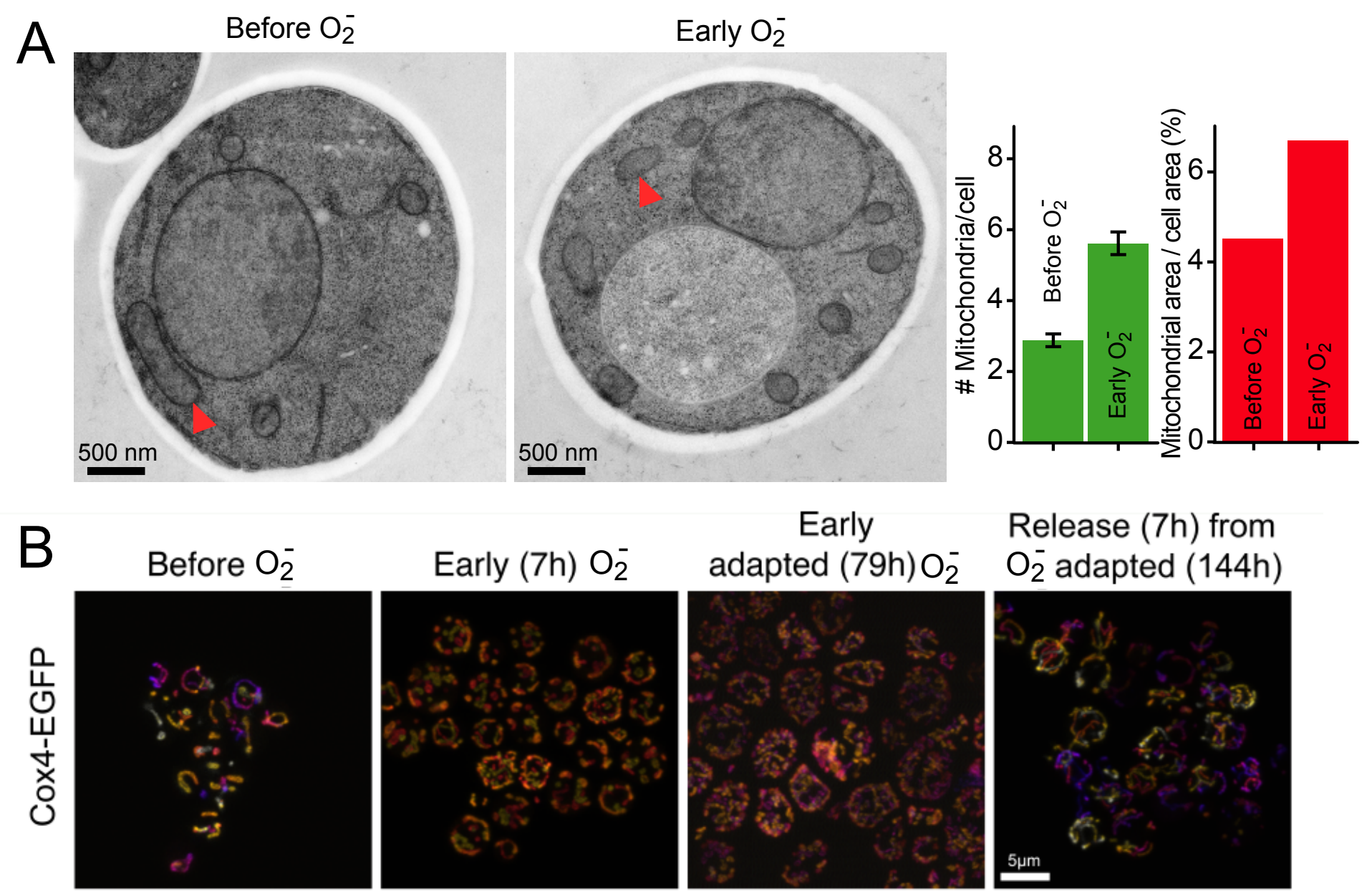

\section{Early}

$$
\text { Early (7h) } \mathrm{O}_{2}^{-}
$$

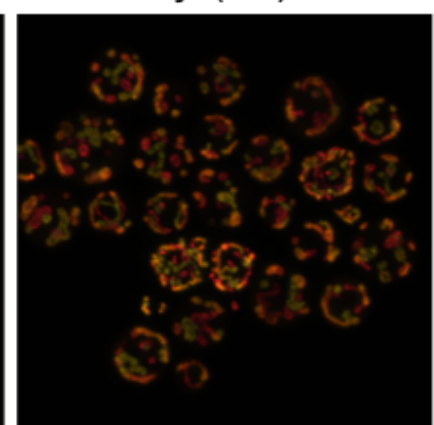
adapted $(79 \mathrm{~h}) \mathrm{O}_{2}^{-}$

Release $(7 \mathrm{~h})$ from
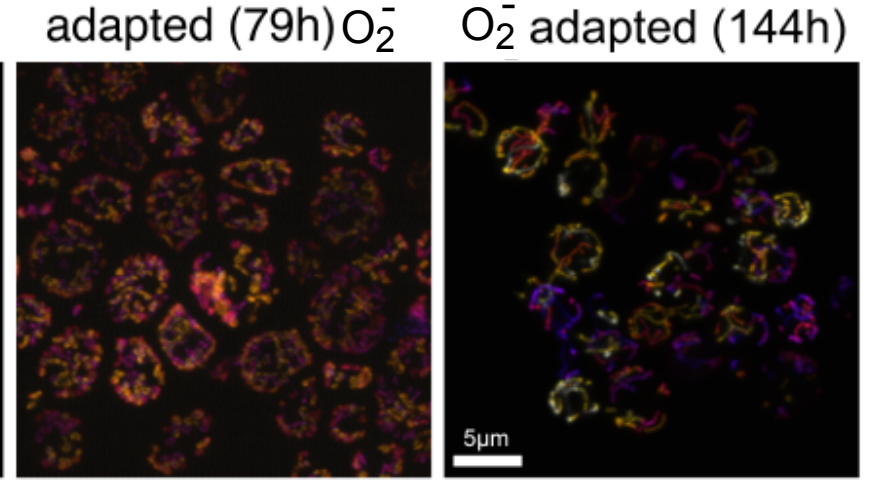

1 Z-slice 18

Figure 2 

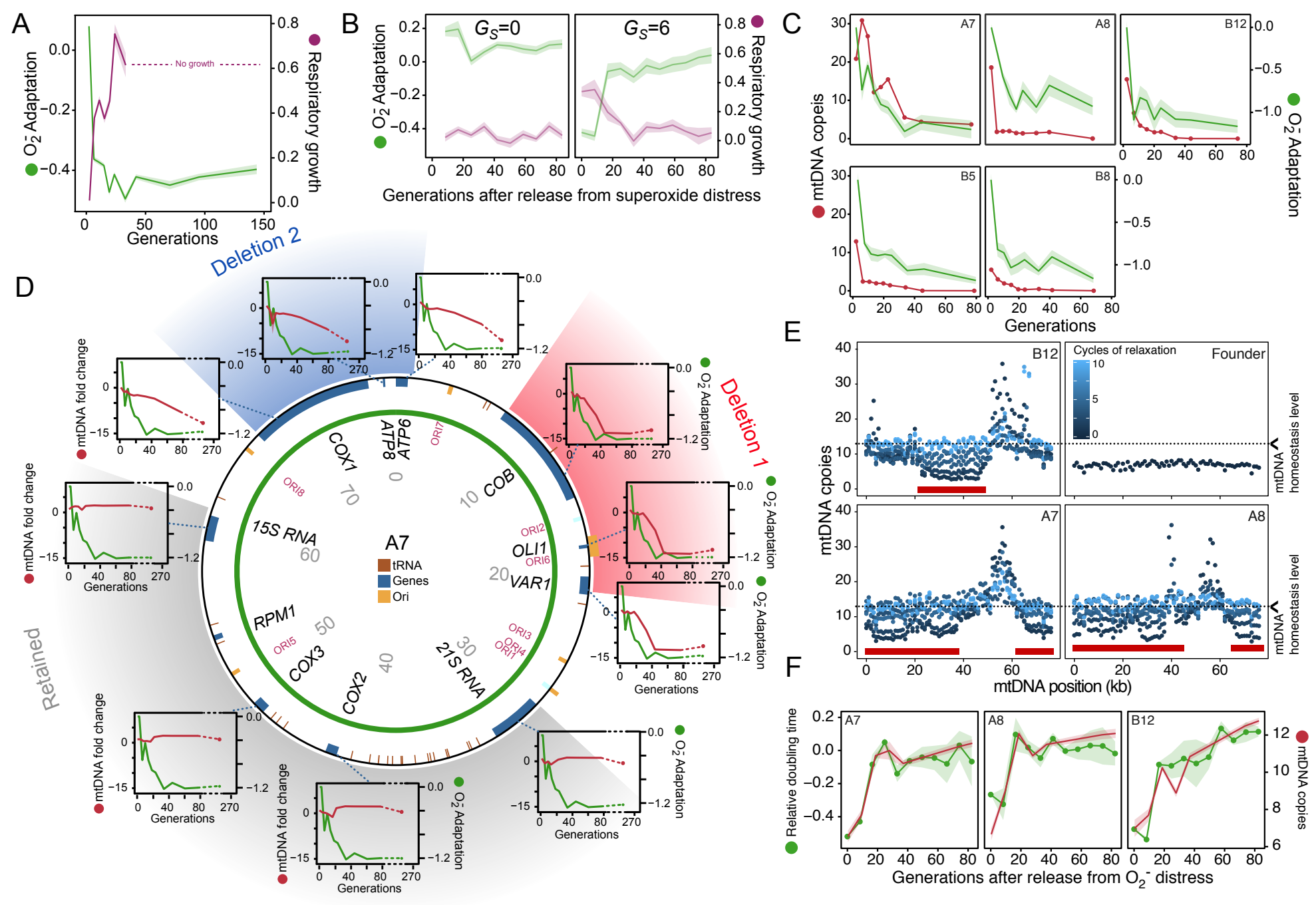

Figure 3 


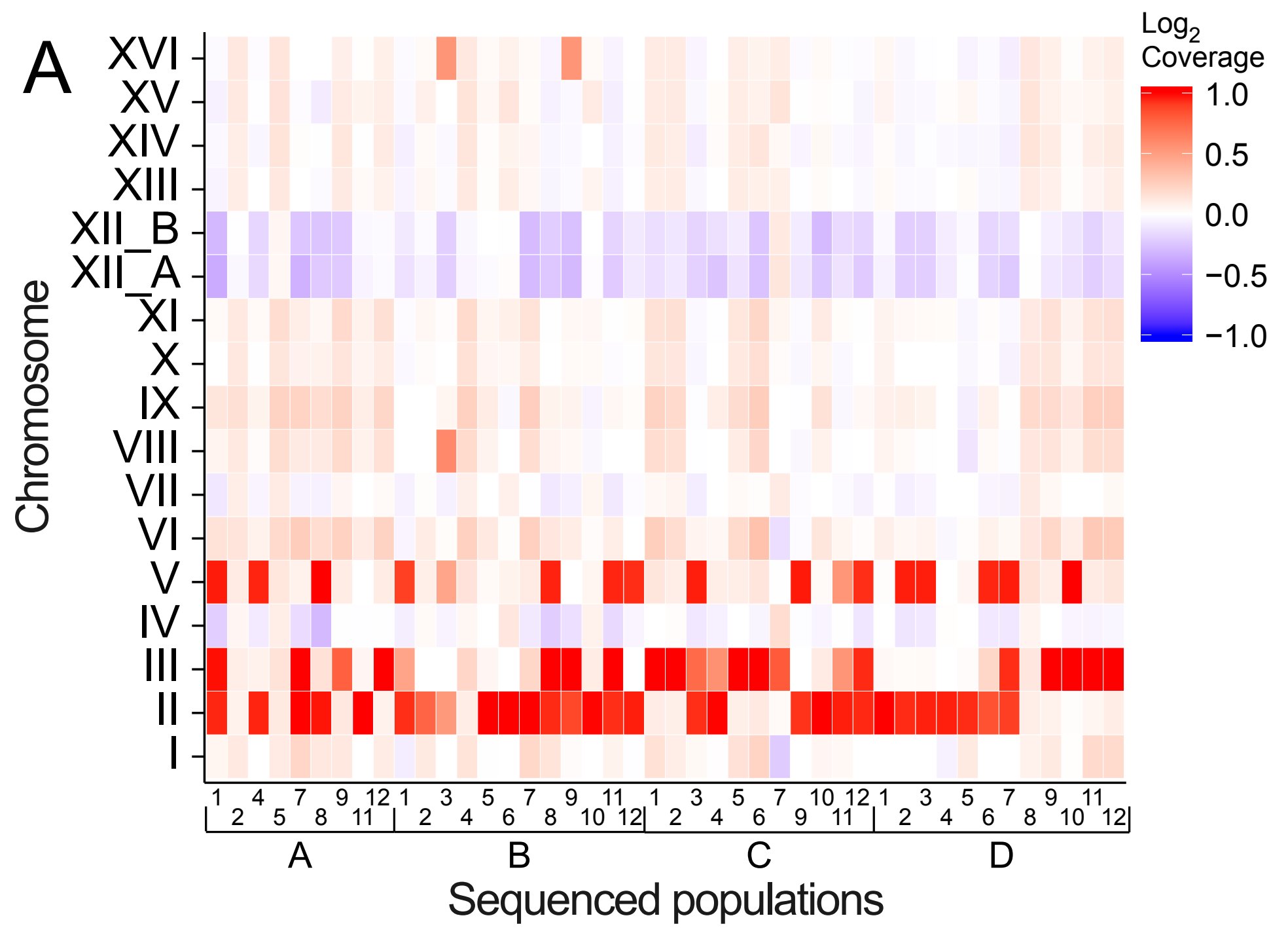

B

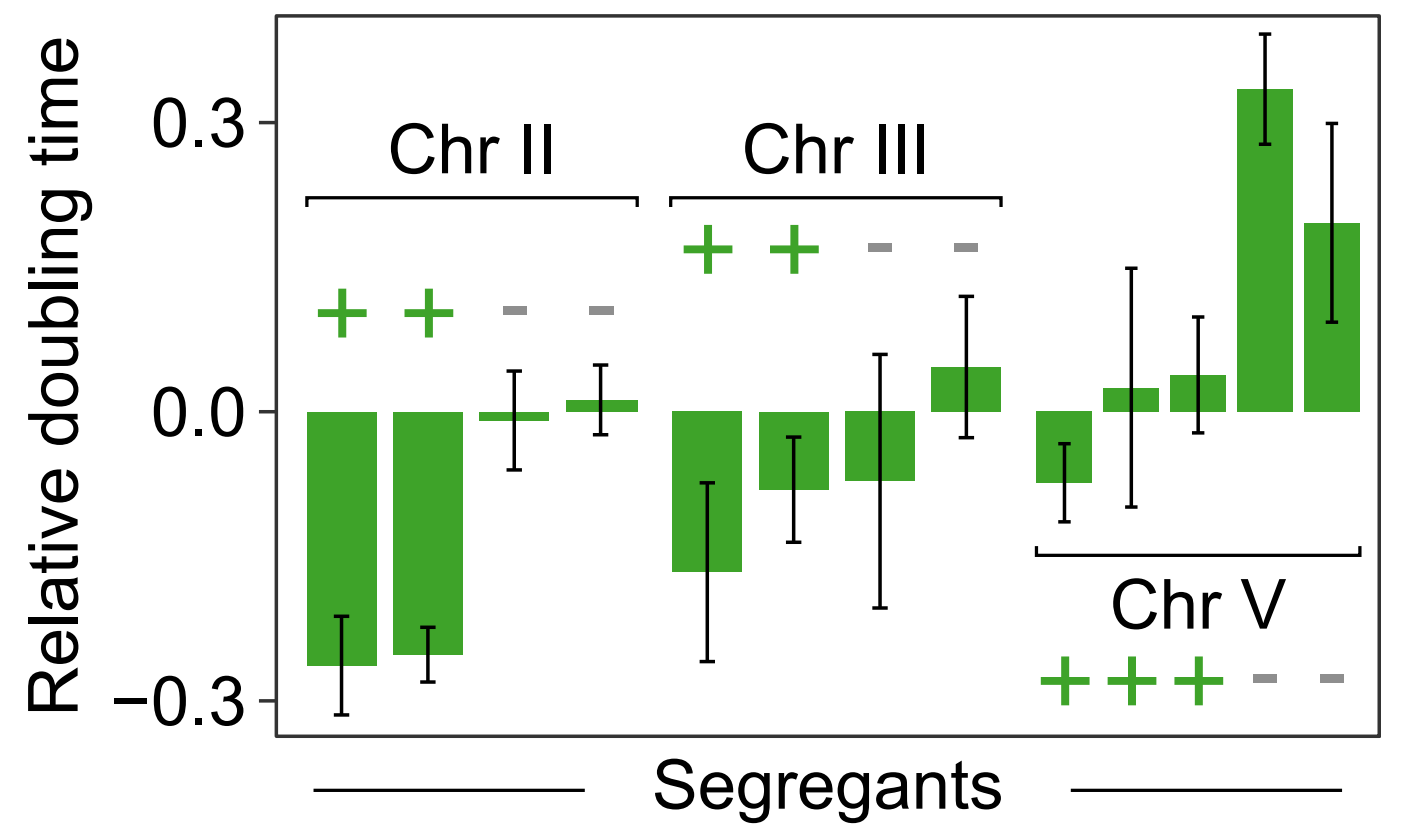

Figure 4 


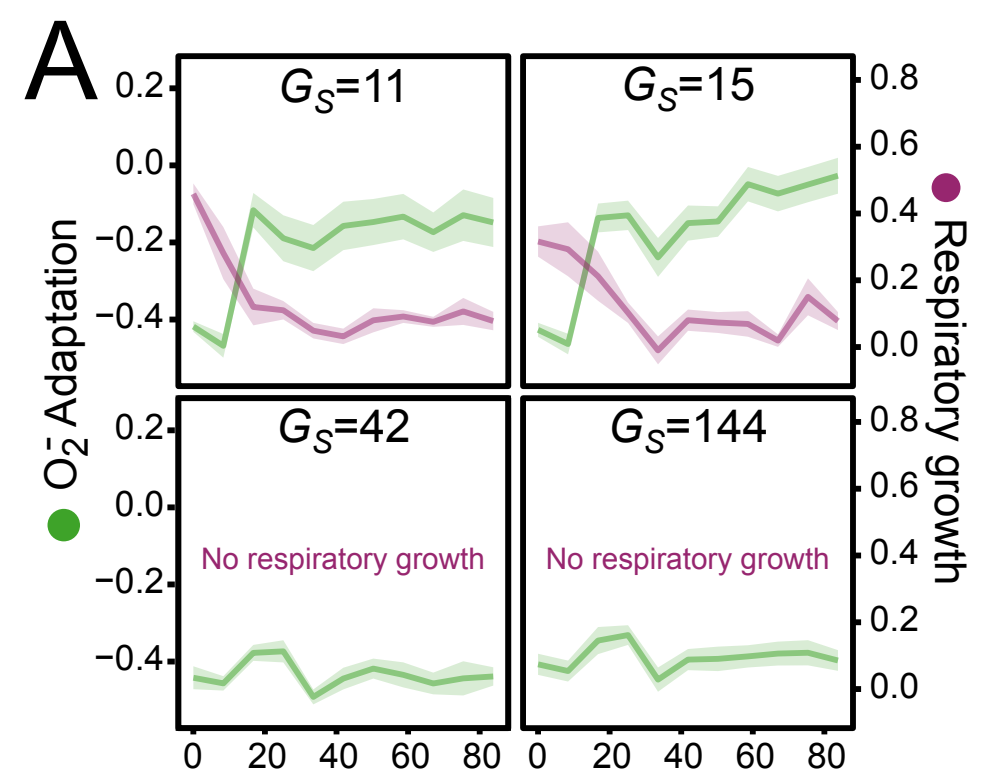

Generations after release from $\mathrm{O}_{2}{ }^{-}$distress

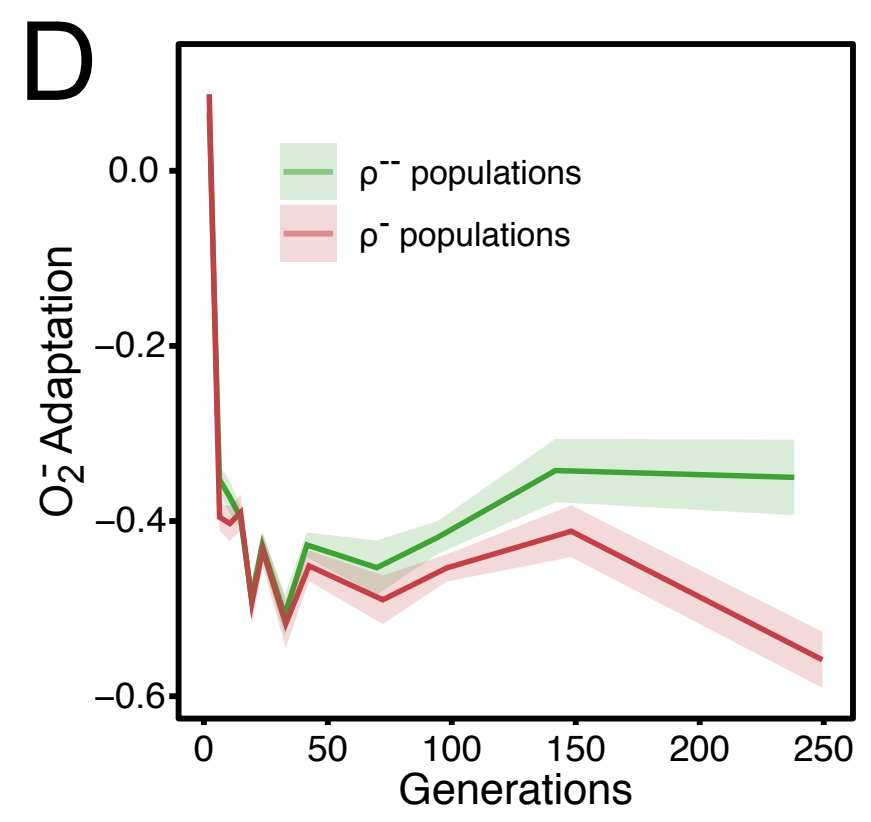

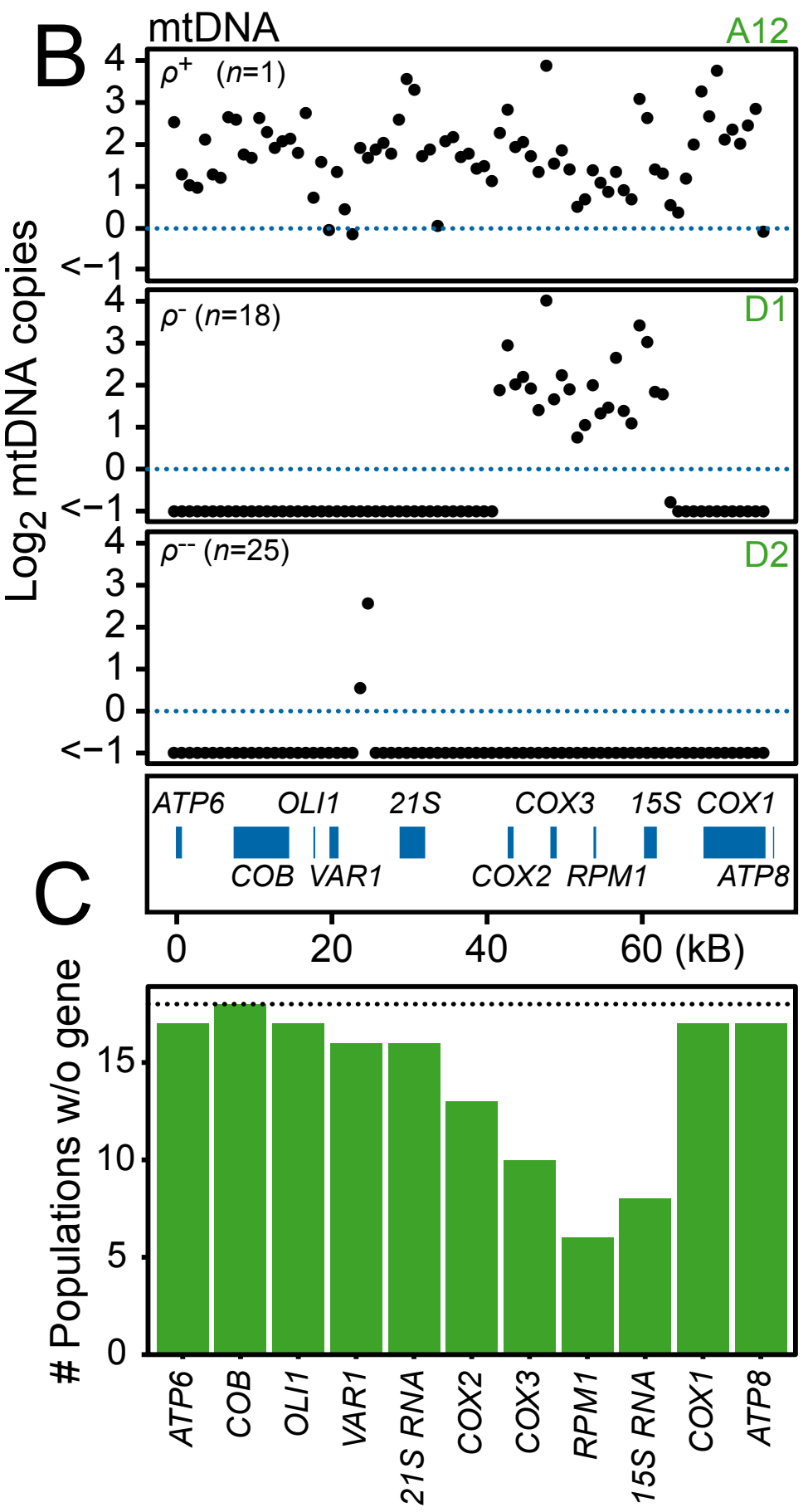




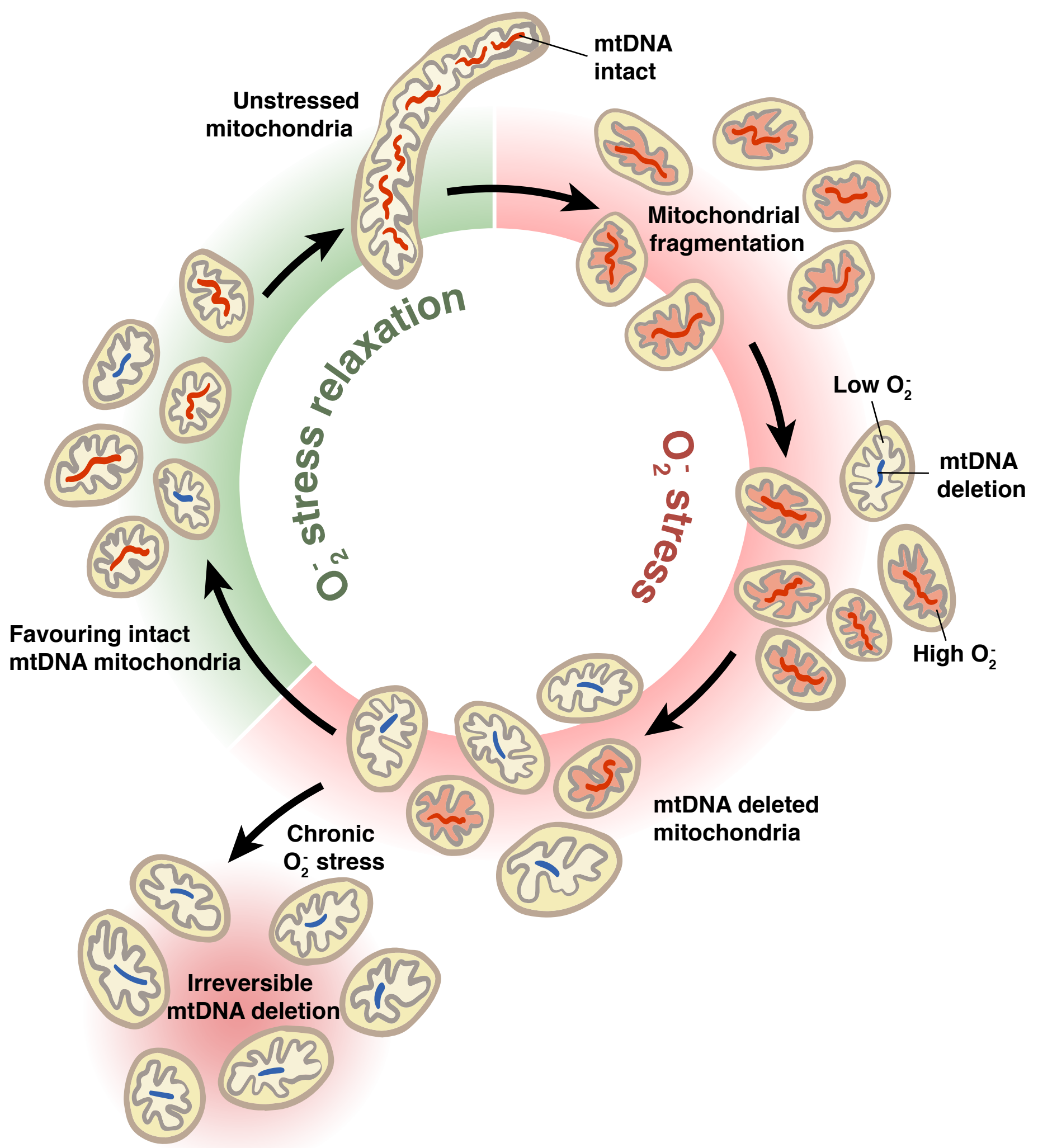

Figure 6 